\title{
ANDRÉ COSTA-MATOS
}

Dados anatômicos preditivos de desfechos cirúrgicos em nefrectomia parcial por tumor: análise prospectiva do escore R.E.N.A.L.

Tese apresentada à Faculdade de Medicina da Universidade de São Paulo para obtenção do título de Doutor em Ciências

Programa de: Urologia

Orientador: Dr. Marcos Francisco Dall'oglio

São Paulo 
Dados Internacionais de Catalogação na Publicação (CIP)

Preparada pela Biblioteca da

Faculdade de Medicina da Universidade de São Paulo

Creprodução autorizada pelo autor

\section{Costa-Matos, André}

Dados anatômicos preditivos de desfechos cirúrgicos em nefrectomia parcial por tumor : análise prospectiva do escore R.E.N.A.L. / André Costa-Matos. -- São Paulo, 2016.

Tese(doutorado)--Faculdade de Medicina da Universidade de São Paulo.

Programa de Urologia.

Orientador: Marcos Francisco Dall’Oglio.

Descritores: 1.Nefrectomia 2.Laparoscopia 3.Neoplasias renais 4.Isquemia 5.Anatomia 6.Classificação 7.Carcinoma

USP/FM/DBD-209/16 
Dedicatória 
À minha família: meus pais, que dedicaram suas vidas ao crescimento dos filhos, à minha esposa Luciana, por me dar a paz necessária ao trabalho, às minhas filhas Laura e Isabela, porque me dão pouco trabalho permitindo o meu estudo, e à Deus, por ter me dado isso tudo. 
Agradecimentos 
Agradeço àqueles que ajudaram na elaboração desse trabalho:

- Aos professores Marcos Dall'Oglio, Alexandre Crippa, José TavaresNeto, Luis Cláudio Correia, Felipe Argolo.

- Aos pesquisadores do ICESP: Enfa. Sanarelly Pires; Valdecir Marvulle;

- Aos preceptors do ICESP: Dr. Roberto Colombo Jr., Dr. Rafael Coelho, Dr. Rodrigo Pessoa, Dr. Matheus Scarpato Chaib; Dr. Daher Chade; Dr. Maurício Cordeiro; Dr. Adriano João Nesrallah; Dr. José Pontes Júnior; Dr. Leopoldo Alves Ribeiro Filho.

- Aos coordenadores da pós-graduação: Prof. Homero Bruschini, Prof. Alberto Azoubel Antunes.

- À Elisa Cruz, secretária da pós-graduação de urologia 
Epígrafe 
II

Learn everything you can, anytime you can, from anyone you can - there will always come a time when you will be grateful you did."

Sarah Caldwell 
Normatização adotada 
Esta tese está de acordo com as seguintes normas, em vigor no momento de sua publicação:

Referências: adaptado de International Committee of Medical Journals Editors (Vancouver).

Universidade de São Paulo. Faculdade de Medicina. Divisão de Biblioteca e Documentação. Guia de apresentação de dissertações, teses e monografias. Elaborado por Anneliese Carneiro da Cunha, Maria Julia de A.L.Freddi, Maria F.Crestana, Marinalva de Souza Aragão, Suely Campos Cardoso, Valéria Vilhena. 3aㅡ ed. São Paulo: Divisão de Biblioteca e Documentação; 2011.

Abreviatura dos títulos e periódicos de acordo com List of Journals Indexed in Index Medicus. 
Sumário 


\section{LISTA DE ABREVIATURAS E SIGLAS}

\section{LISTA DE TABELAS}

LISTA DE GRÁFICOS

LISTA DE QUADROS

LISTA DE FIGURAS

RESUMO

ABSTRACT

1 INTRODUÇÃO

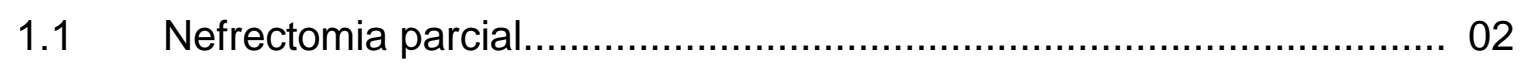

1.2 Nefrectomia parcial videolaparoscópica............................................. 04

$1.3 \quad$ Frequência de nefrectomia parcial.................................................. 06

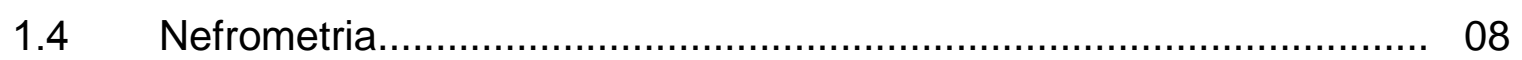

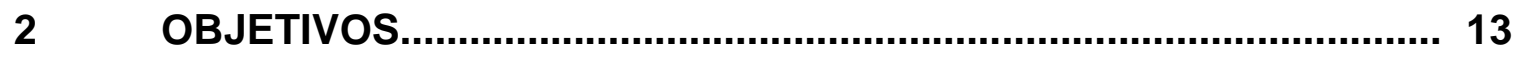

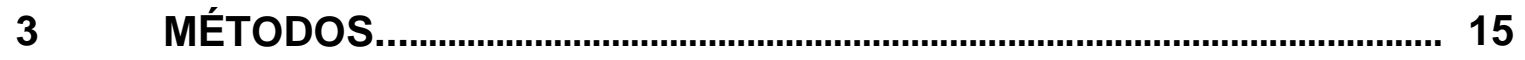

3.1 Estudo piloto......................................................................... 16

3.2 Cálculo do $n$ amostral.................................................................. 18

3.3 Estudo principal....................................................................... 18

3.4 Critérios de inclusão.................................................................. 19

3.5 Critérios de exclusão.................................................................... 19

3.6 Avaliação dos exames de tomografia computadorizada...................... 19

3.7 Descrição do escore RENAL............................................................ 20

3.8 Organização das variáveis e do banco de dados................................. 21

3.9 Indicação da via de acesso................................................................ 22

3.10 Padronização da técnica operatória.................................................. 23

3.11 Tratamento estatístico e teste de hipóteses....................................... 25

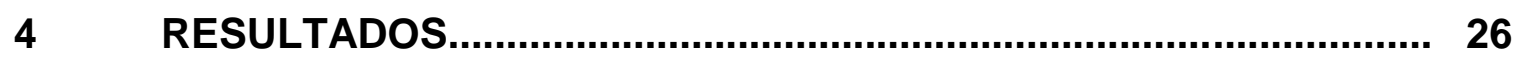

4.1 Descrição da casuística............................................................... 27

$4.2 \quad$ Via de acesso cirúrgica................................................................ 31

4.3 Desfechos intra-operatórios................................................................ 34

4.3.1 Tempo de isquemia.................................................................. 34 
4.3.2 Tempo cirúrgico .................................................................. 36

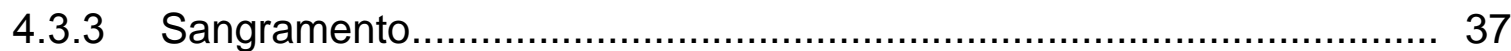

4.4 Análise da influência das variáveis nefrométricas individualmente no desfecho cirúrgico............................................................................ 38

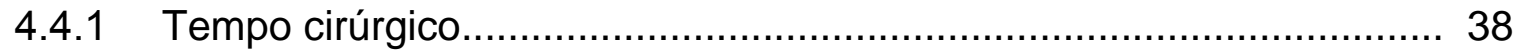

4.4.2 Tempo de isquemia.................................................................. 39

4.4.3 Sangramento........................................................................ 40

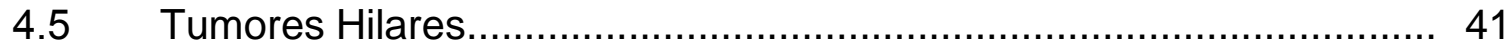

4.6 Complicações pós-operatórias....................................................... 42

4.7 Margens cirúrgicas................................................................... 43

5 DISCUSSÃO.............................................................................. 45

$5.1 \quad$ Desfechos perioperatórios............................................................. 48

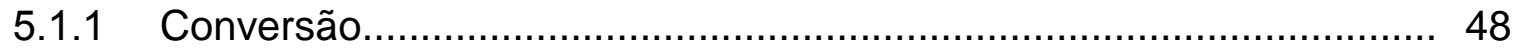

5.1.2 Tempo de cirurgia.................................................................... 50

5.1.3 Tempo de isquemia.............................................................. 51

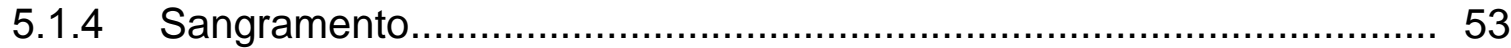

$5.2 \quad$ Margens cirúrgicas.................................................................. 53

5.3 Complicações perioperetórias.......................................................... 54

5.4 Análise crítica das variáveis que compõem o R.E.N.A.L...................... 55

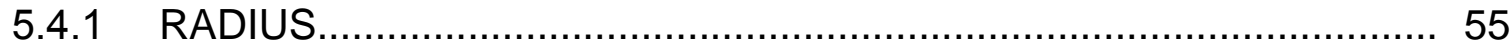

5.4.2 Exophyticus/Endophyticus...................................................... 56

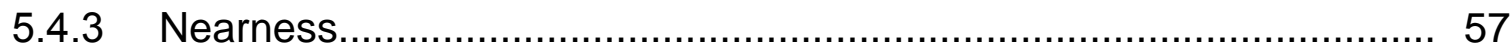

5.4.4 Anterior/Posterior................................................................... 57

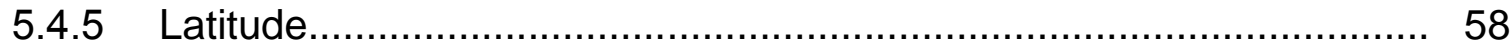

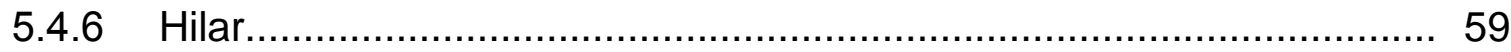

5.4.7 Conclusões acerca da análise crítica das variáveis que compõem o

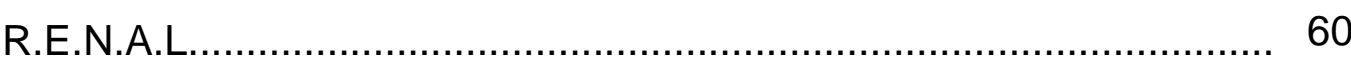


$5.5 \quad$ Limitações do estudo.......................................................... 62

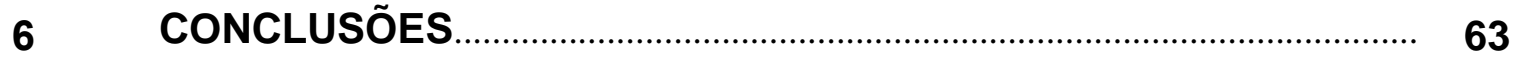

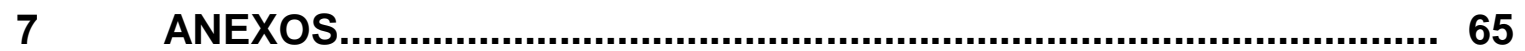

8 REFERÊNCIAS BIBLIOGRÁFICAS........................................... 69 
Gráfico 1 Frequência de nefrectomia de 2002 a 2008 nos EUA........... 07

Gráfico 2 Curva ROC: RENAL x taxa de conversão............................ 33

Gráfico 3 Curva ROC: RENAL x tempo de clapeamento...................... 35

Gráfico 4 Curva ROC entre RENAL e tempo de cirurgia $\geq 180$ min......... 36

Gráfico 5 Sangramento segundo escore RENAL: baixa, média e alta complexidade.................................................................. 37 
Tabela 1 Resultados de nefrectomias parciais para tumores renais unilaterais com rim contralateral normal.

Tabela 2 Avaliação da concordância entre os 2 observadores do escore RENAL - estudo piloto

Tabela 3 RENAL versus tempo de isquemia - Estudo piloto.................... 17

Tabela 4 Motivo da exclusão dos casos do estudo................................. 27

Tabela 5 Características clínicas da população estudada........................ 28

Tabela 6 Características anatômicas e patológicas dos tumores operados

Tabela 7 Distribuição das variáveis conforme escore RENAL................. 30

Tabela $8 \quad$ Via de acesso cirúrgica......................................................... 31

Tabela 9 Frequência de conversão segundo escore RENAL............. 32

Tabela 10 Frequência de conversão segundo escore RENAL $\geq 9 \ldots \ldots \ldots \ldots .32$

Tabela 11 Variáveis perioperatórias e via de acesso cirúrgica.................. 34

Tabela 12 RENAL versus tempo de isquemia renal............................... 35

Tabela 13 Análise univariada dos componentes do RENAL individualmente e tempo de cirurgia..................................... 39

Tabela 14 Análise univariada dos componentes do RENAL individualmente e tempo de isquemia.................................... 40

Tabela 15 Análise univariada dos componentes do RENAL individualmente e sangramento............................................ 41

Tabela 16 Desfechos intra operatórios e localização hilar do tumor renal 42

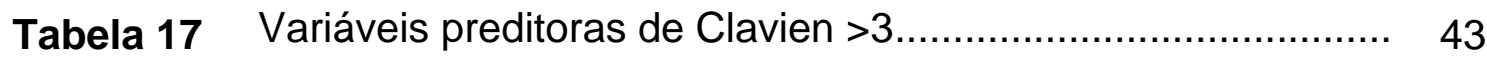


Figura 1 Ilustração do escore RENAL 
Quadro 1 Escore RENAL 
ICESP: Instituto do Câncer do Estado de São Paulo

WIT: Warm ischemia time

OT: Operative time

EBL: Estimated blood loss

LOS: Lengh of stay

ROC curve: Receiving operating caracteristcs

PN: Partial nephrectomy

LPN: Laparoscopic partial nephrectomy

PI: Principal investigator

LC: Low complexity

MC: Medium complexity

HC: High complexity

NPL: Nefrectomia parcial laparoscópica

NPA: Nefrectomia parcial aberta

NTL: Nefrectomia total laparoscópica

NTA: Nefrectomia total aberta

NPR: Nefrectomia parcial robótica

NS: Não significativo

DM: Diabetes mellitus

HAS: Hipertensão arterial sistêmica

IMC: Índice de massa corpórea

EUA: Estados Unidos da América 
Resumo 
Costa-Matos A. Dados anatômicos preditivos de desfechos cirúrgicos em nefrectomia parcial por tumor: análise prospectiva do escore R.E.N.A.L. [tese]. São Paulo: Faculdade de Medicina, Universidade de São Paulo; 2016.

Introdução: A nefrometria tem sido utilizada para avaliação de complexidade anatômica de tumores renais desde 2009. Foram descritos 03 escores para classificar a complexidade anatômica das massas renais: R.E.N.A.L.; PADUA; c-Index. Esses escores foram propostos em casuísticas de nefrectomia parcial (aberta, laparoscópica ou robótica) com caráter retrospectivo, assim fatores de confusão podem explicar os resultados observados na literatura. Objetivo: Testar a acurácia do escore R.E.N.A.L. em predizer desfechos perioperatórios no tratamento cirúrgico de tumors renais $\leq 7,0 \mathrm{~cm}$ em um modelo prospectivo. Métodos: Entre janeiro de 2010 a junho de 2012, 320 pacientes foram submetidos ao tratamento cirúrgico de tumores renais no Instituto do Câncer do Estado de São Paulo. Desses, 173(54,1\%) tinham tumores $\leq 7 \mathrm{~cm}$ e $71(41 \%)$ foram estudados de acordo com os critérios de inclusão e exclusão. Foram avaliados a acurárica do escore R.E.N.A.L. em prever desfechos perioperatórios (tempo de isquemia; tempo operatório; perda sanguínea; taxa de conversão para cirurgia aberta ou totalização da nefrectomia; complicações; tempo de internação e margem cirúrgica), em nefrectomias parciais com uso de curvas ROC, análises uni e multivariadas. Resultados: Nenhum paciente no grupo de baixa complexidade (BC) apresentou tempo de isquemia >20 minutos, contra 12(41,4\%) e $9(64,3 \%)$, respectivamente nos grupos de media complexidade (MC) e alta complexidade ( $A C)(p=0,03)$; porém com acurácia não significativa: $A \cup C=0,643(p=0,07)$. O escore R.E.N.A.L. se associou a taxa de conversão (BC:28,6\%; $M C: 47,6 \% ; A C: 77,3 \%, p=0,02$ ). Pacientes com escore $\leq 8$ foram mais frequentemente submetidos a nefrectomia parcial $(93 \%$ vs. $72 \%, p=0,03)$ e nefrectomia parcial videolaparoscópica $(56,8 \%$ vs. $28 \%$, $p=0,02)$, com boa acurácia: $A \cup C=0,715 ;(p=0,002)$. O escore R.E.N.A.L. também foi associado a tempo operatório. Pacientes com escore $\geq 8$ tiveram 6,06 vezes mais chances de terem tempo cirúrgico $\geq 180 \mathrm{~min}$. $(p=0,017), A \cup C$ de $0,63(p=0,059)$. O escore R.E.N.A.L. não se correlacionou com sangramento, complicações (Clavien >3), tempo de internação ou margem 
cirúgica comprometida. Conclusões: O escore R.E.N.A.L., nesta casuística, mostrou-se ser bom método para prever acesso cirúrgico e tipo de nefrectomia; e também se correlacionou com tempo cirúrgico e de isquemia, porém com acurácias baixas. Entretanto, o escore R.E.N.A.L. não se associou a Clavien $>3$, sangramento, dias de internação ou margens cirúrgicas comprometidas.

Descritores: Nefrectomia; Laparoscopia; Neoplasia renal; Isquemia; Anatomia; Classificação; Carcinoma. 
Costa-Matos A. Accuracy of anatomic data in predict perioperative outcomes in tumor partial nephrectomy: a prospective analisis of R.E.N.A.L. nephrometry score [thesis]. Sao Paulo: "Faculdade de Medicina, Universidade de São Paulo"; 2016

Background and Purpose: The R.E.N.A.L. nephrometry score (RNS) has been validated in multiple open, laparoscopic and robotic partial nephrectomy series. However, those studies are most retrospective and confounding factors could explain the results. The aim of this study was to test the accuracy of RNS in predicting perioperative outcomes in surgical treatment of kidney tumors $\leq 7,0 \mathrm{~cm}(\mathrm{~T} 1 \mathrm{~b})$ in a prospective model. Methods: Between January 2010 and June 2012, 320 patients underwent radical or partial nephrectomy at our institution for the treatment of renal cancer. Of these, $173(54,1 \%)$ patients had a tumor $\leq 7 \mathrm{~cm}, 71$ patients $(41 \%)$ were selected according to the inclusion and exclusion criteria and included in the prospective study. We evaluate the accuracy of the score in predicting perioperative outcomes (WIT, OT, EBL, conversion rate and complications) in partial nephrectomy using ROC curve, univariate and multivariate analyses. Results: No patients in low complexity (LC) group had WIT >20 min, versus $12(41,4 \%)$ and 9(64,3\%) in medium complexity $(\mathrm{MC})$ and high complexity $(\mathrm{HC})$ groups respectively $(\mathrm{p}=0,03)$ however with no significant accuracy: $A U C=0,643 \quad(p=0,07)$. RNS was associated with convertion rate (LC:28,6\%; MC:47,6\%; HC:77,3\%, $\mathrm{p}=0,02$ ). Patients with RNS $\leq 8$ were most often subjected to partial nephrectomy (93\% x $72 \%, p=0,03)$ and laparoscopic partial nephrectomy $(56,8 \% \times 28 \%, p 0,02)$, with good accuracy: $A U C=0,715(p=0,002)$. The RNS was also associated with operative time. Patients with a score $\geq 8$ had 6.06 times greater chance of having a surgery duration $\geq 180$ min. $(p=0,017), \quad A U C=0,63 \quad(p=0,059)$. R.E.N.A.L. score did not correlate with EBL, complications (Clavien $>3$ ), LOS or positive surgical margin. Conclusion: R.E.N.A.L. score, in this data, was a good method in predicting surgical access route and type of nephrectomy. Also was associated with OT and WIT, but with weak accuracy. Although, RNS was not associated with Clavien $>3$, EBL, LOS or positive surgical margin.

Descriptors: Nephrectomy; Laparoscopy; Kidney neoplasms; Ischemia; 
Anatomy; Classification; Carcinoma 
1. Introdução 
O tratamento do câncer de rim baseia-se na extirpação cirúrgica do tumor, que era feita através da nefrectomia radical, primeiramente relatada por Robson há cerca de 50 anos. A experiência acumulada demonstrou que a nefrectomia parcial pode ser tão efetiva quanto a nefrectomia radical no tratamento dessas neoplasias, com as vantagens de preserver função renal e evitar nefrectomias desnecessárias por tumores benignos.

\section{$1.1 \quad$ Nefrectomia parcial}

Apesar de a primeira nefrectomia parcial ter sido relatada por Cnerzy em 1890(1), essa cirurgia só passou a ser realizada de maneira segura e padronizada apenas a partir da década de 80 do século XX e contribuíram para isso os avanços nos métodos de imagem, a maior experiência com cirurgia vascular renal, o melhor entendimento da fisiopatologia da isquemia renal, o aumento na incidência de tumores pequenos e a maior sobrevida dos pacientes(2).

Inicialmente a nefrectomia parcial era indicada para pacientes com risco de insuficiência renal pós nefrectomia total. À medida em que se aumentou a experiência, a nefrectomia parcial foi se popularizando devido aos excelentes resultados oncológicos e passou a ser indicada em situações eletivas. Em 1993, Licht e Novick(3) relataram resultados favoráveis da cirurgia preservadora de néfrons em 241 casos revisados da literatura em pacientes com o rim contralateral normal: os tumores possuíam em média $3,5 \mathrm{~cm}$ e em 
três anos de seguimento houve $95 \%$ de sobrevida. Outros autores(1), relataram seguimento de dez anos de 70 pacientes com 100\% de sobrevida para aqueles com tumores menores que $4 \mathrm{~cm}$. Vários estudos confirmaram a segurança oncológica da nefrectomia parcial (Tabela 1). Esses trabalhos finalmente fizeram que a cirurgia parcial fosse amplamente aceita pelos urologistas e nos levaram à situação atual, com novos procedimentos permitindo intervenções com menor morbidade e melhor qualidade de vida.

Assim, a nefrectomia parcial estabeleceu-se como padrão ouro no tratamento dos tumores renais menores do que $4 \mathrm{~cm}$, e cada vez mais vem sendo aplicada para tumores maiores(2). Deste modo, muitos estudos já mostraram segurança e factibilidade quando aplicada para tumores com até 7cm de diâmetro. Foi publicado em 2010 no Journal of Urology(9), série com seguimento de 7 anos com nefrectomias parciais realizadas em casos com tumores de até $7 \mathrm{~cm}$, tanto pelo procedimento aberto quanto laparoscópico, respectivamente com sobrevida de $97.3 \%$ e $97.5 \%$ livre de metástase. As vantagens para indivíduos com rim único, anatômico ou funcional, são óbvias e inquestionáveis e, nesses casos, admite-se a cirurgia poupadora de néfrons até para casos mais complexos como tumores maiores que $7 \mathrm{~cm}$ ou múltiplos. 
Tabela 1- Resultados de nefrectomias parciais para tumores renais unilaterais com rim contralateral normal

\begin{tabular}{|c|c|c|c|c|}
\hline Séries & $\mathrm{N}$ & SCE (\%) & $\mathrm{RL}(\%)$ & TM \\
\hline Moll et al. (1993)(4) & 98 & 100 & 1 (1 caso) & 4 \\
\hline Herr (1999)(1) & 70 & 97,5 & 1,5 (2 casos) & 3 \\
\hline Hafez et al. (1999)(5) & 45 & 100 & 0 & $<4$ \\
\hline Barbalias et al. (1999)(6) & 41 & 97,5 & 7,3 (3 casos) & 3,5 \\
\hline Filipas et al. (2000)(7) & 180 & 98 & 1,6 (3 casos) & 3,3 \\
\hline Delakas et al. (2002)(8) & 118 & 97,3 & 3,9 (4 casos) & 3,4 \\
\hline Total & 552 & $97,5-100$ & $0-7,3$ & $<4 \mathrm{~cm}$ \\
\hline
\end{tabular}

Contudo, mesmo em indivíduos com função renal adequada e rim contralateral normal, vários estudos(10)(11)(12)(13)(14)(15) demonstram vantagens a longo prazo na preservação da função renal quando se compara a nefrectomia parcial com a radical no tratamento de tumores renais. Martin e Gill em 2002 (10) demonstraram que até mesmo em indivíduos com rim contralateral normal e função renal global preservada, a nefrectomia parcial apresentou vantagens em relação à total na preservação da função renal, medida pelo percentual de aumento da creatinina sérica em um segmento de 5 meses ( $0 \%$ vs. $25 \%, p=0,001)$.

\section{$1.2 \quad$ Nefrectomia parcial videolaparoscópica}

A morbidade perioperatória e, em alguns casos, a dificuldade técnica costumam ser maior na nefrectomia parcial, principalmente quando se aplica a 
via de acesso laparoscópica(16). Por esse motivo, vários estudos comparam resultados obtidos na nefrectomia parcial aberta versus laparoscópica. Gill et al.(16) publicaram em 2003 série com 200 casos de nefrectomias parciais, sendo 100 abertas e 100 por acesso videolaparoscópico; e encontraram dados favoráveis à nefrectomia aberta em relação ao tempo de cirurgia (3 vs 3,9 horas) $(p<0,001)$, perda sanguinea (125 vs. $250 \mathrm{ml})(p<0.001)$ e tempo de isquemia de (27,8 vs 17,5 minutos) ( $p<0.001)$. Quatro anos após, Gill et al.(17), em um estudo multicêntrico reuniram 1.800 casos de nefrectomia parcial. Comparando-se os resultados das 771 videolaparoscópicas com 1028 abertas através de análise multivariada obtiveram menor tempo de cirurgia para nefrectomia laparoscópica $(p<0,0001)$, menor perda sanguínea $(p<0,0001)$ e menor tempo de hospitalização $(p<0,0001)$; entretanto, a nefrectomia laparoscópica foi associada a maior tempo de isquemia $(p<0,0001)$, maior taxa de complicações pos-operatórias (hematuria e fístulas) $(p<0,0001)$, e maior necessidade de procedimentos adicionais $(p<0,0001)$. A função renal em três meses não mostrou-se diferente entre os grupos, com 97.9\% (laparoscópica) e 99.6\% (aberta) das unidades renais recuperando a função. A sobrevida câncer específica em 5 anos, para tumores T1, foi de $99,3 \%$ e $99,2 \%$ para aberta e laparoscópica respectivamente.

Esses trabalhos, entretanto, por terem o caráter retrospectivo, compararam tumores com complexidades diferentes, na maioria das vezes, pareando os grupos apenas pelo tamanho das massas. Sabemos, entretanto, que, muitas vezes, tumores de mesmo volume conferem complexidades cirúrgicas totalmente diferentes, a depender da localização anatômica em 
relação ao parênquima renal ou da proximidade de vasos do hilo, por exemplo. Por falta de padronização com nível I de evidência, a indicação de nefrectomia parcial está sujeita a vieses relacionados à experiência individual do cirurgião, nível de conforto da equipe e outros, inclusive de custos. Por exemplo, um tumor que, por sua localização central, endofítica e próximo a via coletora, pode ser inapropriado para nefrectomia parcial para um determinado cirurgião, mas pode ser factível para outro.

\subsection{Frequência de nefrectomia parcial}

Apesar de segura do ponto de vista oncológico e de apresentar vantagens de sobrevida global e preservação da função renal, a nefrectomia parcial ainda é subutilizada. Patel S. et al.(18) publicaram, em março de 2012, casuística com pacientes submetidos à nefrectomia radical ou parcial por tumores entre 2002 e 2008. Esse trabalho foi baseado no NIS (National Inpatient Sample), um banco de dados que contém $20 \%$ de todas as hospitalizações nos EUA. Conforme o Gráfico 1, publicado no artigo, a proporção de nefrectomias parciais vem aumentando, passou de 15,3\% em 2002 para 24,7\% em 2008, porém ainda é menor do que a frequência de tumores incidentais descobertos atualmente que é por volta de $60 \%(19)(20)$. 


\section{Gráfico 1- Frequência de nefrectomia de 2002 a 2008 nos EUA.}

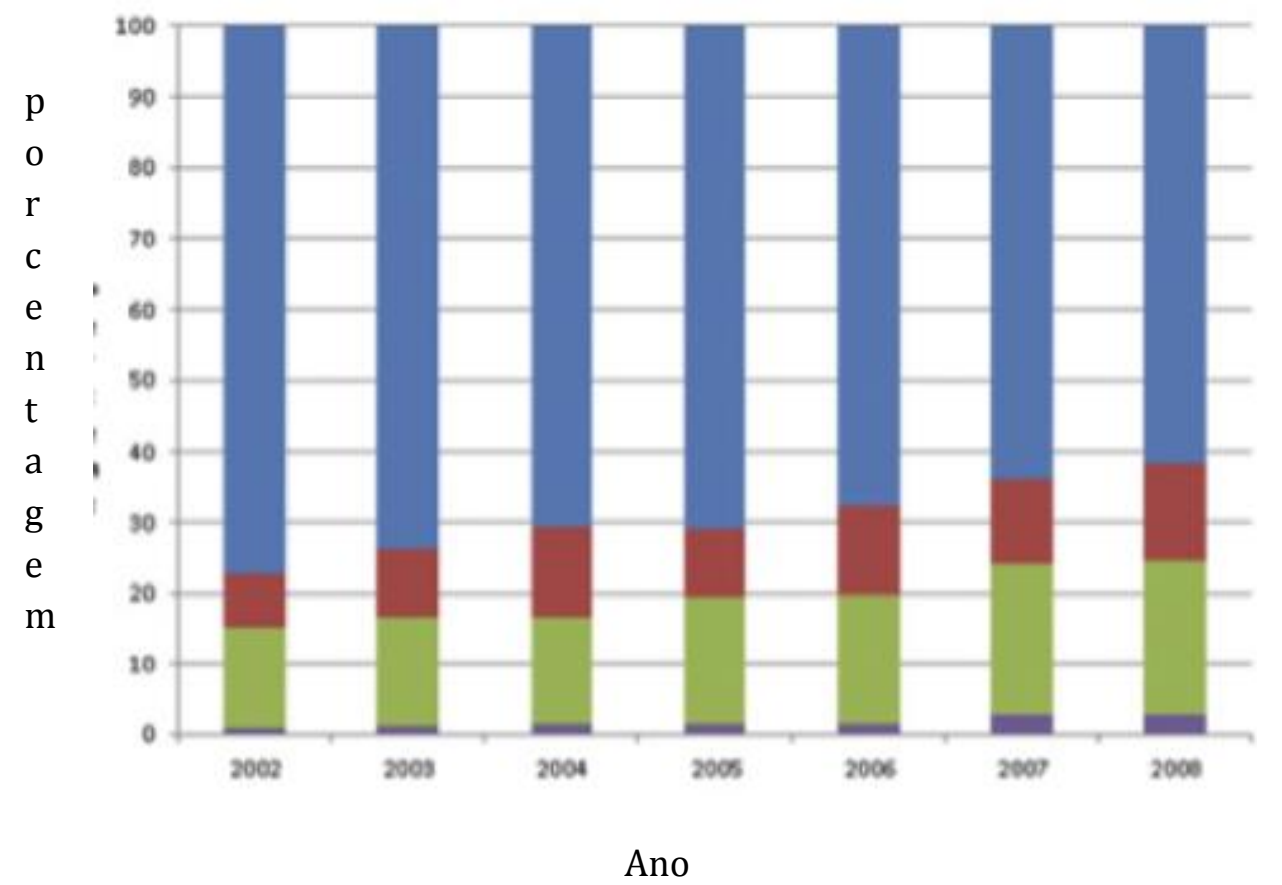

Nefrectomia radical aberta

Nefrectomia radical laparoscópica

Nefrectomia parcial aberta

Nefrectomia parcial laparoscópica

Cabe destacar, na casuística de Patel et al., que a frequência de nefrectomia parcial realizada por videolaparoscopia foi $<5 \%$ em 2008, sobretudo fora das áreas urbanas e relacionadas a hospitais de ensino ou com grande volume cirúrgico. Um trabalho baseado no SEER (Surveillance, Epidemiology and End Results) (21), analisando tumores $<4 \mathrm{~cm}$, observou taxas maiores de nefrectomias parciais que passaram de 20,8\% em 1999 para 45,2\% em 2006. Outra avaliação realizada em centros terciários europeus encontraram aumento de 15\% para 70\% entre 1987 e 2008(22). 
Entretanto, nos últimos 5 anos, a padronização de técnicas de sutura do parênquima renal e a divulgação dos benefícios da cirurgia preservadora de néfrons entre os urologistas, fizeram aumentar a frequência dessa cirurgia. Recentemente, outros trabalhos demonstraram aumento na frequência de nefrectomia parcial em todo o mundo, mas ainda assim acredita-se que essa cirurgia seja subutilizada. Na Europa, uma casuística austro-germânica com 1013 pacientes(23) $(65,6 \%)$ que foram tratados com nefrectomia radical e 560 $(34,4 \%)$ com nefrectomia parcial demonstrou isso. Em 1233 pacientes $(80 \%)$ a cirurgia foi aberta, enquanto $340(22 \%)$ foram tratados por laparoscopia, destes $216(13,7 \%)$ total e $124(7,8 \%)$ parcial. Um estudo coreano relatou aumento anual nas taxas de nefrectomia parcial ao longo dos anos 21,5\% (2008), 38,2\%(2010), 57,2\%(2012), and 66,5\%(2014)(21). Também vem crescendo a frequência de nefrectomia parcial laparoscópica. A chance de ser submetido à nefrectomia parcial ou laparoscópica de 2008 a 2010 em relação a 2000-2001 foi de 6,5 ( $p<0,001)$ e 36 vezes maior $(p<0,001)$, respectivamente $(23)$.

\section{$1.4 \quad$ Nefrometria}

Em 2009 foi publicado por Uzzo et al.(24) um trabalho com o objetivo de padronizar alguns aspectos relacionados à anatomia do tumor renal. Para isso, construíram um escore baseado no grau de complexidade da lesão. Esse escore computou o tamanho do tumor, a relação com o córtex renal (endofítico ou exofítico), a proximidade da via excretora ou do seio renal, localização anterior ou posterior, e a posição do tumor em relação aos polos renais. Também foi conferido um sufixo para os tumores que tinham contato com 
vasos do hilo renal. A partir desses dados, foi confeccionado o escore denominado pelo mnemônico "R.E.N.A.L."(Quadro 1). A cada um dos itens foi atribuído 1 ponto e os sufixos "a" ou "p", para localização anterior ou posterior, e "h" em caso de contato com os vasos do hilo renal. Isso originou três grupos de complexidade: baixa (4 a 6 pontos), média ( 7 a 9 pontos) e alta (10 a 12 pontos). A localização anterior ou posterior não confere complexidade, mas apenas interfere com a via de acesso (lombotomia extraperitoneal, subcostal extra ou transperitoneal, laparoscópica ou retroperitoneoscópica). Por outro lado, o sufixo "h" confere automaticamente maior complexidade. (Quadro e Figura 1.)

\section{Quadro 1- Escore RENAL}

\begin{tabular}{|c|c|c|c|}
\hline & 1 ponto & 2 pontos & 3 pontos \\
\hline $\begin{array}{l}\text { (R)adius (diâmetro } \\
\text { máximo em } \mathrm{cm} \text { ) }\end{array}$ & $\leq 4$ & $>4 \mathrm{e}<7$ & $\geq 7$ \\
\hline (E)xofítico/endofítico & $\geq 50 \%$ & $<50 \%$ & $\begin{array}{l}\text { Totalmente } \\
\text { endofítico }\end{array}$ \\
\hline $\begin{array}{l}\text { (N)nearness } \\
\text { proximidade do } \\
\text { tumor do sistema } \\
\text { coletor ou seio renal } \\
\text { em } \mathrm{mm}\end{array}$ & $7 \mathrm{~mm}$ & $>4 \mathrm{e}<7 \mathrm{~mm}$ & $\leq 4$ \\
\hline (A)nterior/Posterior & \multicolumn{3}{|c|}{ Nenhum ponto atribuído, atribuído a descrição a,p ou x } \\
\hline $\begin{array}{l}\text { (L)ocalização } \\
\text { relativa às linhas } \\
\text { polares }\end{array}$ & $\begin{array}{l}\text { Totalmente } \\
\text { acima da linha } \\
\text { polar superior } \\
\text { ou abaixo da } \\
\text { inferior }\end{array}$ & $\begin{array}{l}\text { Lesões cruzam } \\
\text { a linha polar }\end{array}$ & $\begin{array}{l}>50 \% \text { da massa } \\
\text { cruza a linha } \\
\text { polar (a) ou cruza } \\
\text { a linha média do } \\
\text { rim (b) ou esta } \\
\text { totalmente entre } \\
\text { as linhas polares } \\
\text { (c) }\end{array}$ \\
\hline
\end{tabular}




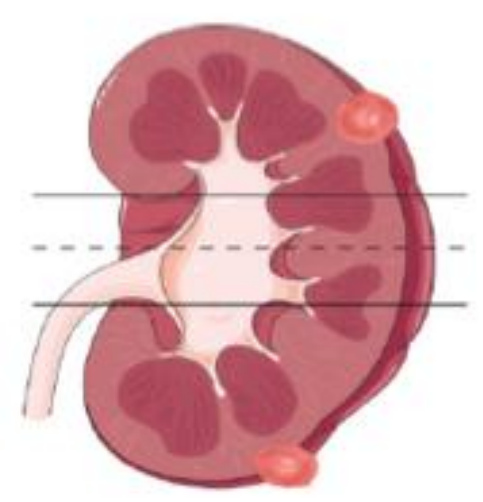

1

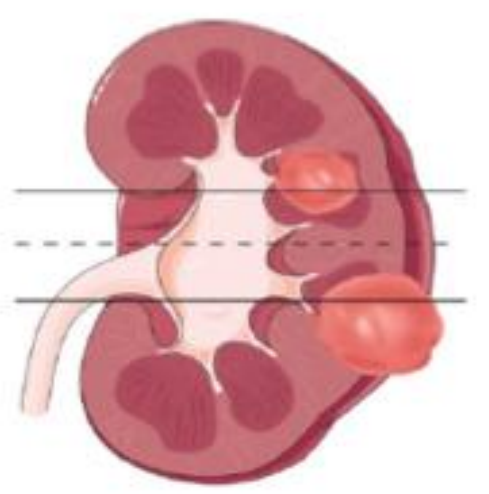

2

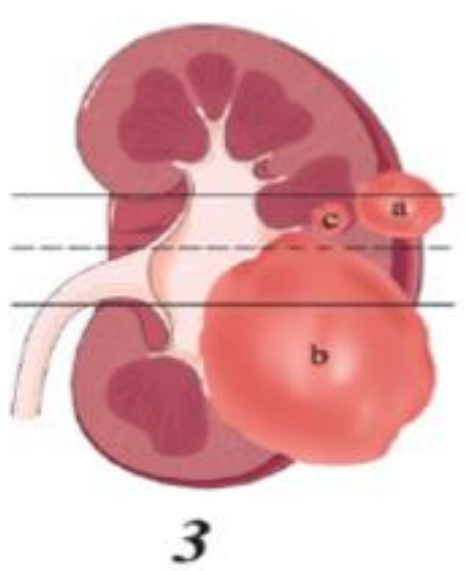

Figura 1- Ilustração do escore RENAL

Outras tentativas de graduar a complexidade anatômica dos tumores renais têm surgido na literatura, como o escore PADUA(25) em 2009, e o Cíndex(26) em 2010. O primeiro atribuiu pontos às características anatômicas e é muito semelhante ao RENAL, o segundo é um índice de centralidade do tumor, que baseia-se na relação entre a distância entre o tumor e o centro do rim e o raio do tumor. Entretanto, o escore RENAL é o mais utilizado em nosso meio provavelmente por ter sido descrito por influentes autores norteamericanos.

Outros estudos foram desenvolvidos por diversos grupos, utilizando o escore RENAL. Kolla et al. (27) avaliaram a concordância interobservador, em que o escore foi aplicado a 51 pacientes por 3 médicos distintos, obtendo-se um alto índice de concordância. Matthew Hayn et al.(28) estudaram o mesmo escore em 141 nefrectomias parciais videolaparoscópicas realizadas por um mesmo cirurgião, e encontraram diferença significativa entre os grupos de baixa, média e alta complexidades, no que se referiu a tempo de isquemia, 
perda sanguínea e tempo de hospitalização. Alexander Kutikov et al.(29), aplicaram o escore a 525 nefrectomias e encotraram correlação com grau e subtipo histológico. Entretanto, todos os estudos mencionados anteriormente foram retrospectivos ou baseado em banco de dados preexistentes.

Embora existam críticas a esse escore, atualmente é a maneira mais objetiva e reprodutível de se comparar os tumores renais, no que diz respeito à complexidade anatômica. Uma das críticas é que o peso atribuído a cada variável é o mesmo, porém sabemos que a influência de cada uma delas pode ser diferente. A influência nos desfechos do tamanho da lesão, por exemplo, pode ser maior do que sua localização em relação aos polos renais, assim tumores com níveis de complexidade diferentes podem ser agrupados conjuntamente. O próprio Uzzo et al. no artigo original(24) discutiram alguns desses aspectos relacionados à fragilidade do escore. Um tumor com somatória $4 a(1+1+1+a+1)$ seria menos complexo do que outro com iguais características, porém localizado entre os polos $(1+1+1+a+3$ : $6 a)$. Por isso, os autores sugeriram que a descrição do escore seja feita separadamente, item por item, por exemplo, um tumor de $6 \mathrm{~cm}$, exofítico, a $1 \mathrm{~cm}$ do sistema coletor, anterior e polar seja descrito como: $2+1+1+A+1$. Assim, discriminando cada um dos itens se teria melhor avaliação da complexidade da lesão. Contudo, os estudos publicados com o escore consagraram a exposição dos valores já somados, deixando assim tumores diferentes no mesmo grupo.

Enfim, a proposta dos autores é de que a nefrometria poderia ser usada na padronização de estudos, como preditor de desfechos perioperatórios e na seleção da via de acesso cirúrgica. A literatura atual 
demonstra que a frequência de nefrectomia parcial vem aumentando nos últimos anos(21), quer pela maior frequência de diagnósticos de tumores em estágios iniciais, quer pelo aumento da experiência dos cirurgiões com nefrectomia parcial. Com isso as complicações perioperatórias relacionadas a este procedimento, que são maiores do que na nefrectomia radical, vem aumentado. Entretanto, o único estudo randomizado que comparou nefrectomia parcial versus total, não demonstrou diferença em relação à sobrevida global, sugerindo que em indivíduos com rim contralateral normal, a retirada total do rim doente não é um procedimento proibitivo e, se eventuais complicações forem previstas, talvez seja a melhor indicação(30). Isso justifica este estudo, para avaliar os parâmetros anatômicos que possam prever desfechos em nefrectomia por tumor e, assim, melhorar as perspectivas e qualidade de vida dos pacientes. 
2. Objetivos 
Analisar dados anatômicos relacionados ao tumor renal (escore RENAL) como preditores de desfechos cirúrgicos em nefrectomia parcial.

\section{Principal}

- 1 - Escore RENAL pode prever tempo de isquemia $>20$ minutos

\section{Secundários}

1. Correlacionar o escore RENAL e seus componentes individualmente com:

a. tempo de cirurgia;

b. sangramento;

c. fístulas urinárias;

d. hematúria;

e. taxa de conversão para cirurgia aberta (no caso das laparoscópicas);

f. taxa de totalização da nefrectomia;

g. taxa de complicação;

h. margens cirúrgicas. 
3. Métodos 


\subsection{Estudo piloto}

Inicialmente foi realizado um estudo piloto retrospectivo com o objetivo de padronizar a coleta dos dados que compõem o escore RENAL e estimar o $n$ amostral para a hipótese principal a ser testada.

Este estudo piloto avaliou retrospectivamente a associação entre o escore RENAL e desfechos perioperatórios em nefrectomia parcial. A hipótese principal testada foi:

- $\mathrm{H} 1$ - Escore RENAL pode prever tempo de isquemia $>20$ minutos

Nessa revisão de prontuários, encontrou-se 140 nefrectomias parciais operadas no ICESP (Instituto do Câncer do Estado de São Paulo) e ICHC (Instituto Central do Hospital das Clínicas de São Paulo) entre junho de 2008 e novembro de 2010, das quais 39 foram incluídas de acordo com os critérios de seleção e por conterem os dados necessários à realização das análises de desfechos perioperatórios.

O escore RENAL foi avaliado por 2 observadores independentes (1 radiologista e 1 urologista) e testado a sua concordância entre eles. Obteve-se um índice Kappa 0,9 ( $p<0,001)$, (Tabela 2). 
Tabela 2- $\quad$ Avaliação da concordância entre os 2 observadores do escore RENAL - estudo piloto

\begin{tabular}{l|c|c|c}
\hline \multirow{2}{*}{ RENAL: Observador 1 } & \multicolumn{3}{|c}{ RENAL: Observador 2 } \\
\cline { 2 - 4 } & Baixa complexidade & Média complexidade & Alta complexidade \\
\hline Baixa complexidade & $6(100,0 \%)$ & 0 & 0 \\
\hline Média complexidade & $1(5,3 \%)$ & $18(94,7 \%)$ & 0 \\
\hline Alta complexidade & 0 & $1(12,5 \%)$ & $7(87,5 \%)$ \\
\hline Kappa 0,9 $(\mathrm{p}<0,001)$. & & &
\end{tabular}

O escore RENAL foi estratificado conforme proposto por Uzzo et al. em 3 graus de complexidade: baixa, moderada e alta. A partir disto foram correlacionados com as variáveis perioperatórias. Este estudo exploratório demonstrou que o escore RENAL se correlacionou com menor tempo de isquemia $(14 ; 19,4$ e 25,0 minutos $-p=0,06)$ e sangramento $(145 ; 250$ e 390 ml) $(p=0,02$ e 0,006$)$ intra-operatório no grupo de baixa, média e alta complexidades respectivamente. Foi encontrada a proporção de $36 \%$ de indivíduos que tiveram tempo de isquemia renal $>20$ minutos nos grupos de baixa e média complexidades versus $89 \%$ no grupo de alta complexidade, (Tabela 3).

Tabela 3- RENAL versus tempo de isquemia - Estudo piloto.

\begin{tabular}{lcc}
\hline RENAL & \multicolumn{2}{c}{ Tempo de Isquemia } \\
\hline \multirow{2}{*}{ Baixa } & $<20 \mathrm{~min}$ & $\geq 20 \mathrm{~min}$ \\
Media & $4(100 \%)$ & 0 \\
Alta & $14(64 \%)$ & $8(36 \%)$ \\
$\mathrm{P}=0,004$ & $1(11 \%)$ & $8(89 \%)$ \\
\hline
\end{tabular}




\subsection{Cálculo do $\mathrm{n}$ amostral}

Foi de 30 casos o número total de pacientes necessários para demonstrar a diferença de $67 \%$ no tempo de isquemia entre indivíduos com escore RENAL de baixa e média complexidades em relação ao de alta complexidade, com erro alfa de $5 \%$ e poder do teste de $80 \%$, mas, para aumentar o poder do teste, o $\mathrm{n}$ amostral mínimo foi de 50 pacientes.

\subsection{Estudo principal}

O estudo iniciou-se em janeiro de 2010, com a coleta prospectiva de dados dos pacientes com tumores renais de até $7,0 \mathrm{~cm}$, de acordo com os critérios de seleção. A inclusão de novos casos terminou em junho de 2012. O paciente entrava no estudo a partir da indicação de extirpação cirúrgica do tumor renal, momento em que era explicado o termo de consentimento livre e esclarecido (TCLE - Anexo II) previamente aprovado pelo Comitê de Ética em Pesquisa (Parecer Anexo I) do Hospital das Clínicas da USP (parecer $n^{\circ}$ 0027/11), e depois era acompanhado por 90 dias após a cirurgia para avaliação de desfechos pós-operatórios. Aos pacientes que aceitassem participar da pesquisa era solicitada uma tomografia do Serviço de Radiologia do ICESP. Essa era a única intervenção realizada nos indivíduos, necessária para a adequada padronização do escore. 


\subsection{Critérios de inclusão}

- Lesão sólida ou cística (Bosniak III ou IV11) com captação de contraste em rim com indicação de nefrectomia parcial: tumor de até $7,0 \mathrm{~cm}$ de diâmetro, independente da localização anatômica.

- Possuir exame de imagem (tomografia ou ressonância magnética) no sistema interno do hospital, que permitisse a visualização completa das imagens em cortes axiais, sagitais e coronais.

\subsection{Critérios de exclusão}

- Pacientes com único rim anatômico ou funcional;

- Pacientes com insuficiência renal crônica (Creatinina $>1,5 \mathrm{mg} / \mathrm{dl}$ );

- Pacientes com múltiplos tumores renais: mais de 01;

- Pacientes submetidos a cirurgias prévias no rim ou em andar superior do abdome ipsilateral ao tumor renal;

- Pacientes com calculose renal ipsilateral ao tumor;

- Pacientes com tuberculose renal;

- Pacientes que não concordarem em assinar o termo de consentimento.

\subsection{Avaliação dos exames de tomografia computadorizada}

As tomografias foram realizadas em aparelhos PHILIPS de 16, 40 ou 64 canais e o escore RENAL classificado pelo pesquisador principal. A

\footnotetext{
1 Bosniak MA. The current radiological approach to renal cysts. Radiology. 1986;158:1-10
} 
visualização das imagens foram feitas através do programa da PHILIPS disponível nos computadores do ICESP.

Cada indivíduo foi avaliado clinicamente, laboratorialmente, através de exames de rotina pré-operatória, e radiologicamente com tomografias de abdome e pelve e radiografia de tórax.

\subsection{Descrição do escore RENAL:}

R: Raio - Maior diâmetro do tumor - <=4cm; 4-7cm;>7cm - são atribuídos 1,2 e 3 pontos respectivamente

E: Exofítico/Endofítico - predominantemente exofítico (>50\%), predominantemente endofítico (50\%), totalmente endofítico (envolvido totalmente por parênquima renal normal) - são atribuídos 1,2 e 3 pontos respectivamente.

N: Nearness - Proximidade do sistema coletor ou do seio renal. $>7 \mathrm{~mm}$; 7 4mm; <4mm distantes do sistema coletor ou seio renal. São atribuídos 1,2 e 3 pontos respectivamente.

A: Anterior/posterior - Localização primariamente anterior ou posterior do tumor. A essa variável não são atribuído pontos, mas apenas a letra "A" ou "P" ou "X" (quando não é possível determinar se o tumor é anterior ou posterior).

L: Localização - Localização do tumor em relação à linha polar. A linha polar é uma linha que passa perpendicularmente à junção entre o parênquima renal e o seio renal na borda medial do rim. Os tumores podem se localizar totalmente 
fora das linhas polares, cruzar a linha polar ou estarem predominantemente $(>50 \%)$ entre as linhas polares ou cruzar a linha média axial do rim. São atribuídos 1,2, 3 e 3 pontos respectivamente.

A soma desses parâmetros nefrométricos pode variar de 4 a 12, sendo considerados com escore baixo entre 4 e 6 (BC), intermediário entre 7 e 9 (MC) e complexo entre 10 e 12 (AC). Esses grupos foram criados conforme proposto por Kutikov e Uzzo(24) a partir dos resultados obtidos em 50 nefrectomias realizadas no serviço em que o trabalho original que descreveu o escore foi realizado. Os casos de baixa e média complexidade foram passiveis de nefrectomia parcial, na maioria das vezes, por técnica minimamente invasiva, e os casos de alta complexidade foram submetidos à nefrectomia parcial aberta ou total videolaparoscópica. Esse somatório é acompanhado da letra que define a localização anterior "a", posterior "p" ou indefinida " $x$ " do tumor. Também foi conferido o sufixo "h" se a lesão encosta nos vasos do hilo renal.

\subsection{Organização das variáveis e do banco de dados}

Os cirurgiões que participavam dos procedimentos preenchiam 0 protocolo da pesquisa que posteriormente era digitado em um banco de dados por enfermeiras contratadas para esse fim. Após isso, os dados eram conferidos pelo pesquisador principal e transferidos para sistema SPSS versão 17.0. 
Foram avaliados idade, sexo, IMC (índice de massa corpórea: Peso/Altura ${ }^{2}$ ), índice de comorbidades de Charlson (31)(Charlson, 1987), história de tabagismo, hipertensão arterial sistêmica e diabetes melitus, presença de sintomas relacionados ao tumor (dor, hematúria, massa palpável, presença de síndrome paraneoplásica), presença de metástase, via de acesso cirúrgica, tempo de cirurgia, tempo de clampeamento do pedículo renal, congelamento, abertura da via coletora, sangramento, necessidade de hemotransfusão, taxa de conversão para cirurgia aberta e totalização e os respectivos motivos, creatinina pré e pós-operatória (primeiro pós-operatório e dois meses após), TFG (calculada pela fórmula de Krokroft \& Gault²), presença de fístulas urinárias, sangramento ou hematúria pós-operatória, escala de complicação de Clavien(32) - avaliada até o pós-operatório 90 dias - e dados anatomopatológicos.

\subsection{Indicação da via de acesso}

Inicialmente, para todos os pacientes era proposta a nefrectomia parcial laparoscópica, considerada, neste protocolo de pesquisa, padrão ouro de conduta. Qualquer conduta diferente desta foi considerada como conversão. Após a cirurgia, os pacientes eram classificados de acordo com a operação efetivamente realizada: nefrectomia parcial/enucleação

\footnotetext{
${ }^{2}$ Homem: Clearence de Creatinina $=[((140$-Idade $) \times$ Peso $(\mathrm{kg})) /($ Creatinina Sérica $\mathrm{x} 72)]$

Mulher: Clearence de Creatinina $=[((140$-Idade $) \times$ Peso $(\mathrm{kg})) /($ Creatinina Sérica $\times 12)] \times 0,85$
} 
videolaparoscópica (NPL); nefrectomia parcial/enucleação aberta (NPA); nefrectomia total videolaparoscópica (NTL); ou nefrectomia total aberta (NTA). Eram consideradas conversões preemptivas aquelas que ocorriam antes do início do procedimento e intra-operatórias as que ocorriam durante o mesmo.

\subsection{Padronização da técnica operatória}

\section{Nefrectomia parcial/enucleacão videolaparoscópica - NPL:}

Paciente em decúbito latetal oposto ao lado operado, inserção de 3 a 4 portais laparoscópicos, acesso transperitoneal com mobilização do cólon, dissecção do pedículo renal e remoção da fascia de gerota e da gordura perinefrética com identificação o tumor e demarcação da linha de secção do parênquima renal com bisturi elétrico. A isquemia era realizada com clampeamento da artéria renal ou da artéria e da veia. Eram administrados $1 \mathrm{ml} / \mathrm{kg}$ de Manitol a 20\%, 5 minutos antes da oclusão vascular e após o desclampeamento. O tumor era enucleado usando-se tesoura fria tentando-se deixar uma margem mínima de segurança. Não era realizado exame de congelação da margem. A hemostasia e fechamento do sistema coletor eram feitos com sutura contínua na área cruenta do rim com Vicryl® 2.0 agulha SH (Johnson \& Johnson New Brunswick, NJ, USA). Após, uma sutura externa em "U" ancorada com Hem-o-lok® Ligation System (Teleflex Incorporated, Limerick, PA USA) com aproximação das bordas do parênquima renal era realizada com Vicryl® 0 agulha CT. 


\section{Nefrectomia parcial/enucleação aberta - NPA}

A cirurgia aberta era realizada através das incisões lombares ou subcostais. O procedimento operatório seguia conforme descrito anteriormente na laparoscopia. Para a sutura do parênquima renal eram aplicados pontos hemostáticos em "U" ou "X", sem uso de Hem-o-lok.

\section{Nefrectomia total videolaparoscópica - NTL}

O procediemento era inicialmente semelhante ao que fora descrito para a nefrectomia parcial, porém o pedículo renal e o ureter eram ligados com Hem-o-lok e o rim dissecado do retroperitônio juntamente com a gordura perinefrética (nefrectomia radical).

$\underline{\text { Nefrectomia total aberta - NTA }}$

Semelhante à nefrectomia radical videolaparoscópica, porém através das incisões subcostal ou lombar. 


\subsection{Tratamento estatístico e teste de hipóteses}

\section{Comparação entre as variáveis}

Para comparação entre duas ou mais médias foram utilizados o teste $t$ de Student e a análise de variância (ANOVA) respectivamente, medianas o Mann Whitney e entre proporções o qui-quadrado, a correção para continuidade ou o teste exato de Fisher, quando se aplicava.

Foram desenhadas curvas ROC (Receiver Operating Characteristic), para avaliar a acurácia do escore RENAL em prever desfechos intraoperatórios (tempo de isquemia, sangramento, tempo cirúrgico) e o índice de Youden para identificar o ponto de corte com a melhor performance em sensibilidade e especificidade

Foi avaliado a influência individual de cada variável componente do escore RENAL no desfecho cirúrgico através de regressão linear e logística em modelos uni e multivariados.

Foram considerado estatisticamente significante valores de $p<0,05$. 
4. Resultados 


\subsection{Descrição da casuística}

Entre janeiro de 2010 e junho de 2012 foram operados 320 indivíduos para câncer de rim no Instituto do Câncer do Estado de São Paulo - ICESP. Desses, 173 (54\%) tinham tumores de até $7 \mathrm{~cm}$. Foram selecionados para análise dos dados, de acordo com os critérios de inclusão e exclusão 71 pacientes. Os 102 indivíduos excluídos com os respectivos motivos estão listados na Tabela 4.

Tabela 4- Motivo da exclusão dos casos do estudo

\begin{tabular}{lc}
\hline Rim único & 8 \\
IRC & 17 \\
Tuberculose renal & 3 \\
Pielonefrite & 1 \\
Cirurgia renal prévia & 7 \\
Cirurgia abdominal prévia & 11 \\
Calculose renal ipsilateral ao tumor & 12 \\
Não possuir tomografia do ICESP & 43
\end{tabular}

A idade média foi de $60 \pm 12,7$ anos, 39 (54,9\%) eram do sexo masculino. A Tabela 5 resume as características clínicas da população estudada. 
Tabela 5- Características clínicas da população estudada

\begin{tabular}{lc}
\hline Gênero & $39(55 \%)$ \\
$\quad$ Masculino & $32(45 \%)$ \\
Idade, anos & $60 \pm 12,7(22-88)$ \\
IMC & $27,6 \pm 4,5(17,9-39,6)$ \\
\hline ASA & $5(7 \%)$ \\
I & $60(84,5 \%)$ \\
II & $6(8,5 \%)$ \\
III & $32(45,1 \%)$ \\
Hipertensão arterial sistêmica & $9(12,7 \%)$ \\
Diabetes melitus & $19(26,8 \%)$ \\
Tabagismo & \\
Charlson & $40(56,3 \%)$ \\
$\leq 3$ & $31(44,3 \%)$ \\
$>3$ & $54(76,1 \%)$ \\
\hline Incidental & $1,0 \pm 0,84$ \\
\hline Creatinina pré-operatória (mg/dl) & $13,7 \pm 1,3$ \\
Hemoglobina pré-operatória (mg/dl) & \\
\hline
\end{tabular}

O escore RENAL mediano foi de 9 (4-11). A tabela 6 resume as características dos tumores operados. 
Tabela 6- Características anatômicas e patológicas dos tumores operados

\begin{tabular}{lc}
\hline Tamanho do tumor & $4,1 \pm 1,4(1,3-7,0)$ \\
RENAL (mediana - min-max ) & $9(4-11)$ \\
\hline RENAL & $7(9,9 \%)$ \\
Baixa complexidade (4-6) & $42(59,2 \%)$ \\
Média complexidade (7-9) & $22(31 \%)$ \\
Alta complexidade (10-12) & \\
\hline Tipo histológico & $34(47,9 \%)$ \\
Células claras & $9(12,7 \%)$ \\
Papilar & $6(8,5 \%)$ \\
Cromófobo & $4(5,6 \%)$ \\
Medular & $2(2,8 \%)$ \\
Oncocitoma & $6(8,5 \%)$ \\
Angiomiolipoma & $10(14 \%)$ \\
Outros & \\
& \\
\hline Fuhrman & $8(11,3 \%)$ \\
1 & $35(49,3 \%)$ \\
2 & $17(23,9 \%)$ \\
3 & $2(2,8 \%)$ \\
4 & $9(12,7 \%)$ \\
não classificável & \\
\hline Estágio patológico & \\
T1a & $39(54,9 \%)$ \\
T1b & $21(29,6 \%)$ \\
T2 & $6(8,5 \%)$ \\
T3a & $5(7,0 \%)$ \\
\hline Estudo da margem cirúrgica & \\
Livres & \\
Coincidentes & \\
Focalmente comprometidas & $50(70,4 \%)$ \\
Comprometidas & $17(23,9 \%)$ \\
\hline
\end{tabular}


Correlacionou-se os grupos de baixa, moderada e alta complexidades segundo o escore RENAL com as variáveis perioperatórias. Vide tabela 7.

Tabela 7- Distribuição das variáveis conforme escore RENAL

\begin{tabular}{|c|c|c|c|c|}
\hline & $\mathrm{BC}$ & $\mathrm{MC}$ & $\mathrm{AC}$ & $\mathrm{P}$ \\
\hline$A S A>2$ & $1(14,3 \%)$ & $4(9,5 \%)$ & $1(4,5 \%)$ & NS \\
\hline BMI & 27,7 & 27,6 & 27,6 & NS \\
\hline Charlson $<3$ & $6(85,7 \%)$ & $22(85,7 \%)$ & $18(85,4 \%)$ & NS \\
\hline Tempo de internação (dias) & 3,6 & 3,9 & 4,2 & NS \\
\hline Tempo cirúrgico (min) & 134 & 163 & 185 & $\begin{array}{c}<0,05-\text { (entre os } \\
\text { grupos de BC e } \\
\text { AC) }\end{array}$ \\
\hline Sangramento (ml) & 376 & 460 & 347 & NS \\
\hline Tempo de isquemia $(\mathrm{min})^{*}$ & 10 & 15 & 20 & 0,01 \\
\hline Clavien $\geq 3$ & 0 & $7(16,6 \%)$ & $1(4,5 \%)$ & NS \\
\hline Incidental & $7(100 \%)$ & $33(78,6 \%)$ & $14(63,6 \%)$ & 0,05 \\
\hline Margens positivas & $1(14,3 \%)$ & $2(4,8 \%)$ & $1(4,5 \%)$ & 0,17 \\
\hline Margens coincidentes & $1(14,3 \%)$ & $7(16,7 \%)$ & $9(40,9 \%)$ & $\begin{array}{c}0,04 \text { (entre os } \\
\text { grupos de } B C \text { e } \\
A C)\end{array}$ \\
\hline Estágio $\geq T 2$ & 0 & $6(15,4 \%)$ & $5(23,8 \%)$ & 0,05 \\
\hline Células claras & $2(28,6 \%)$ & $17(44,7 \%)$ & $15(68,2 \%)$ & $\begin{array}{c}0,05 \text { (entre os } \\
\text { grupos de } B C \text { e } \\
A C \text { ) }\end{array}$ \\
\hline Laparoscopia & $5(71,4 \%)$ & $22(52,4)$ & $5(22,7 \%)$ & 0,01 \\
\hline Conversão & $2(28,6 \%)$ & $20(47,6 \%)$ & $17(77,3 \%)$ & 0,02 \\
\hline Resfriamento & 0 & 0 & $4 / 16$ & \\
\hline Totalização & 0 & $5(11,9)$ & $6(27,3 \%)$ & 0,05 \\
\hline
\end{tabular}

Os tumores de alta complexidade foram mais sintomáticos $(36,4 \%)$ do que os de menor complexidade (0), $p=0,05$, foram de estágio maior ( $23,8 \%$ vs. $0, p=0,05)$, do tipo células claras $(68,2 \%$ vs. $28,6 \%, p=0,05)$, tiveram menor 
margem de ressecção cirúrgica $(40,9 \%$ vs. $14,3 \%, p=0,04)$, foram menos operados por laparoscopia $(22,7 \%$ vs. $71,4, p=0,01)$ e tiveram maiores taxas de conversão e totalização.

\subsection{Via de acesso cirúrgica}

Quarenta $(56,4 \%)$ pacientes foram operados por laparoscopia e 31 $(43,6 \%)$ através de cirurgia aberta (Tabela 8$)$. A maioria dos tumores de baixa complexidade $(71,4 \%)$, aproximadamente metade dos de média $(52,4 \%)$, e a minoria dos de alta $(22,7 \%)$ foram operados por laparoscopia, $p<0,01$.

Tabela 8- Via de acesso cirúrgica

\begin{tabular}{lc}
\hline & $\mathrm{N}(\%)$ \\
\hline Nefrectomia parcial videolaparoscópia & $32(45,1 \%)$ \\
Nefrectomia parcial aberta & $28(39,4 \%)$ \\
Nefrectomia total videolaparoscópica & $8(11,3 \%)$ \\
Nefrectomia total aberta & $3(4,2 \%)$ \\
\hline
\end{tabular}

Dos 71 pacientes incluídos, $26(36,6 \%)$ tiveram conversão preemptiva para cirurgia aberta (2 NTA 24 NPA). Quarenta e cinco iniciaram o procedimento por laparoscopia, 8 foram convertidos para NTL e 5 para cirurgia aberta (4 NPA e 1 NTA). A tabela 9 demonstra a frequência de conversão segundo escore RENAL. 
Tabela 9- Frequência de conversão segundo escore RENAL.

\begin{tabular}{lccc}
\hline RENAL & NPL & Outras & $p$ \\
\hline BC & $5(71,4 \%)$ & $2(28,6 \%)$ & 0,02 \\
MC & $22(52,4 \%)$ & $20(47,6 \%)$ & \\
AC & $5(22,7 \%)$ & $17(77,3 \%)$ & \\
\hline
\end{tabular}

A acurácia do RENAL em prever taxa de conversão foi testada através da curva ROC que demonstrou uma AUC de $0,715(0,595-0,836)(p=0,002)$ (gráfico 1). Avaliando-se um ponto de corte privilegiando a especificidade da informação, encontramos para RENAL $\geq 9,41 \%$ de sensibilidade e $90,3 \%$ de especificidade.

Pacientes com RENAL $<9$ foram mais frequentemente submetidos a NP $(93 \% \times 72 \%, p=0,03)$ e NPL $(56,8 \%$ x 28\%, p 0,02) Tabela 10.

Tabela 10- Frequência de conversão segundo escore RENAL $\geq 9$

\begin{tabular}{lccc}
\hline RENAL & NPL-VLP & Outros & $p$ \\
\hline$<9$ & $25(56,8 \%)$ & $19(43,2 \%)$ & 0,02 \\
$\geq 9$ & $7(28 \%)$ & $18(72 \%)$ & \\
\hline
\end{tabular}


Gráfico 1- Curva ROC: RENAL x taxa de conversão

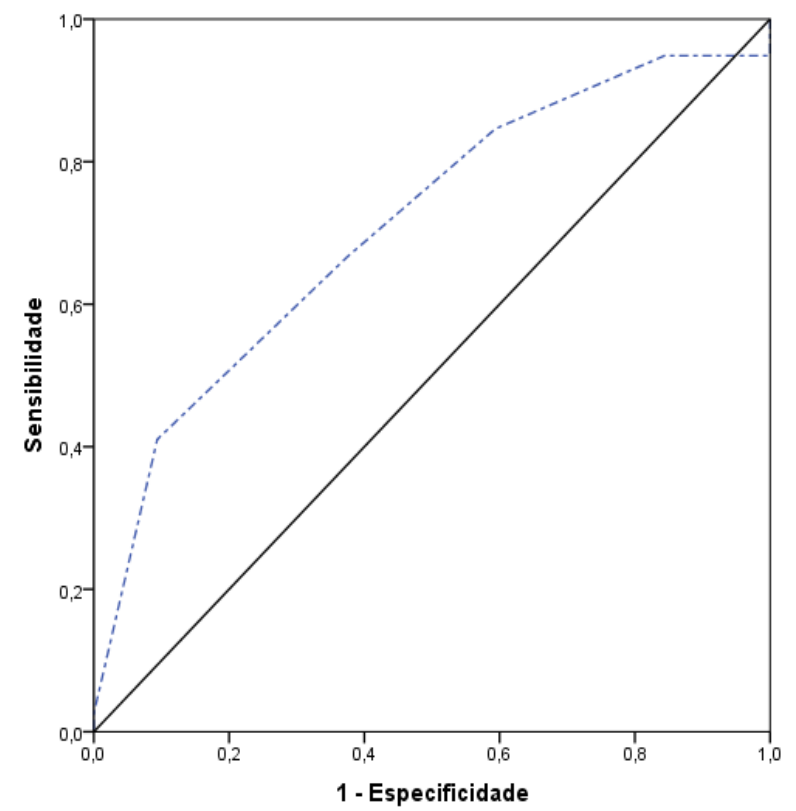

AUC: $0,715(0,595-0,836)$

$\mathrm{p}=0,002$

A via de acesso cirúrgica não influenciou os desfechos perioperatórios, (Tabela 11). 
Tabela 11- Variáveis perioperatórias e via de acesso cirúrgica

\begin{tabular}{|c|c|c|c|c|c|}
\hline & NPL & NPA & NTL & NTA & $p$ \\
\hline $\begin{array}{l}\text { TC média } \\
(95 \% \mid C)\end{array}$ & $\begin{array}{c}174 \pm 51(90- \\
300)\end{array}$ & $\frac{155 \pm 50(70-}{261)}$ & $\begin{array}{c}189 \pm 77(120- \\
354)\end{array}$ & $133 \pm 38(90-160)$ & NS \\
\hline $\begin{array}{l}\text { TI mediano } \\
(95 \% \mathrm{IC})\end{array}$ & $17(10-35)$ & $11,5(8-30)$ & - & - & NS \\
\hline $\begin{array}{l}\text { Sangramento } \\
\text { média }(95 \% \mid \mathrm{C})\end{array}$ & $376(223-529)$ & $491(318-662)$ & $293(123-465)$ & $513(-/ 1597)$ & NS \\
\hline $\begin{array}{l}\text { Temp. } \\
\text { internação } \\
\text { média(min-max) }\end{array}$ & $3,6+1,1(1-6)$ & $4,3+1,2(3 / 7)$ & $4,1+2,2(2 / 9)$ & $3,7+1,2(3-5)$ & NS \\
\hline Clavien $>3$ & $3(9,4 \%)$ & $3(10,7 \%)$ & & & \\
\hline
\end{tabular}

\subsection{Desfechos intra-operatórios}

\subsubsection{Tempo de isquemia}

O tempo mediano de isquemia aumentou conforme os graus de complexidade dos tumores segundo o escore RENAL (BC: 10 minutos; MC: 15 minutos; $A C: 20$ minutos $-p=0,01)$.

Admitimos que um tempo de clampeamento do hilo renal de até 20 minutos é considerado de baixo risco para lesão renal permanente. Vinte e um pacientes tiveram tempo de isquemia $>20$ minutos. Encontramos maior proporção de indivíduos com tempo de isquemia $>20$ minutos nos grupos de 
média e alta complexidades, e nenhum no grupo de baixa complexidade, (Tabela 12).

Tabela 12- RENAL versus tempo de isquemia renal

\begin{tabular}{lccc}
\hline Complexidade & $<20 \min \mathrm{n}(\%)$ & $\geq 20 \min \mathrm{n}(\%)$ & $\mathrm{p}$ \\
\hline $\mathrm{BC}$ & $4(100)$ & 0 & $0,03 \mathrm{LR}$ \\
$\mathrm{MC}$ & $17(58,6)$ & $12(41,4)$ & \\
$\mathrm{AC}$ & $5(35,7)$ & $9(64,3)$ & \\
\hline
\end{tabular}

A curva ROC desenhada entre RENAL e tempo de clampeamento encontrou uma $A \cup C=0,643(0,503-0,784),(p=0,07)$. Avaliando-se um ponto de corte privilegiando a especificidade da informação, encontramos que quem tem escore $\geq 9$ tem $28,6 \%$ de sensibilidade e $81,6 \%$ de especificidade de ter tempo de clampeamento > 20min., (Gráfico 2).

\section{Gráfico 2- Curva ROC: RENAL x tempo de clapeamento}

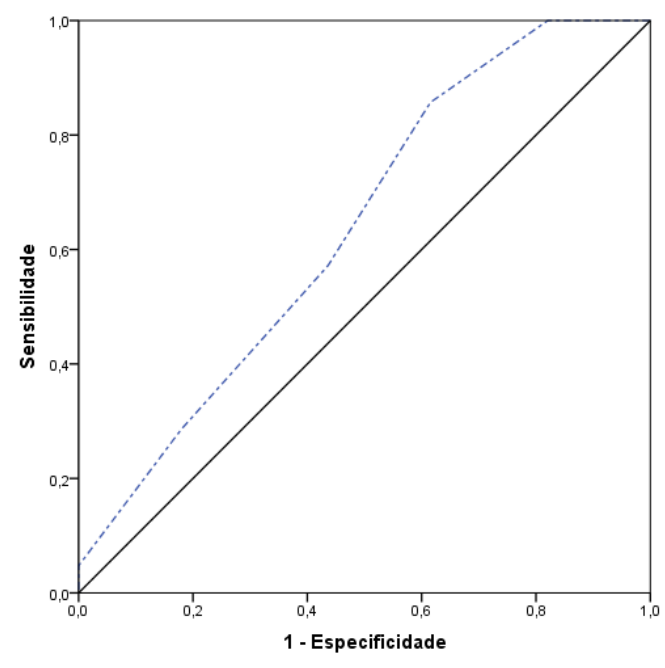




\subsubsection{Tempo cirúrgico}

A média de tempo cirúrgico dos indivíduos aumentou conforme os graus de complexidade dos tumores segundo o escore RENAL (BC: 134 minutos; $\mathrm{MC}$ : 163 minutos; $A C$ : 185 minutos $-p<0,05$, entre os grupos $B C x$ $A C)$.

Considerou-se um tempo cirúrgico $\geq 180$ minutos, como prolongado. A curva ROC demonstrou que o escore RENAL conseguiu prever tempo de cirurgia prolongado com uma AUC de 0,63 ( $p=0,059)$. Utilizando-se o Índice de Youden para prever o melhor ponto de corte, encontramos RENAL $\geq 8$ como preditor de tempo de cirurgia $\geq 180$ minutos, com sensibilidade de $89,3 \%$ e especificidade de 37,2\%, (Gráfico 4).

Gráfico 2- Curva ROC entre RENAL e tempo de cirurgia $\geq 180 \mathrm{~min}$

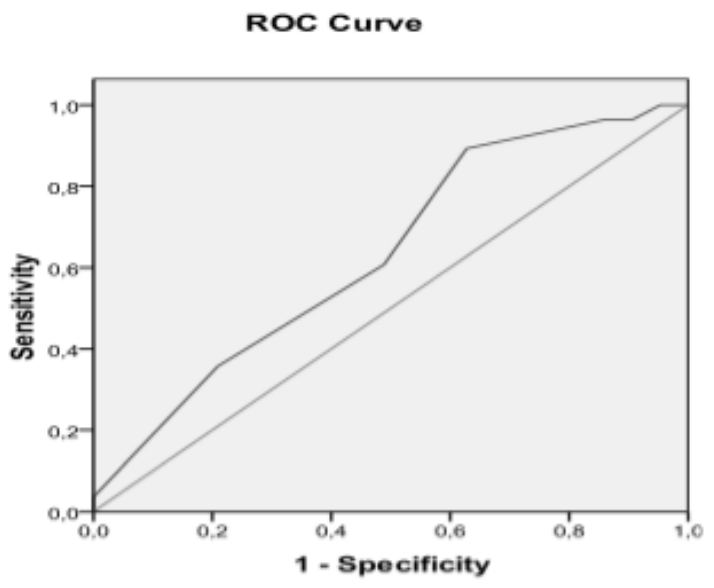

$A \cup C=0,63 ; p=0,059$ 
Fazendo análise de risco relativo segundo Mantel-Haenszel, encontramos que quem tem escore $\geq 8$ tem chance de 4,94 vezes mais de ter tempo de cirurgia $\geq 180$ minutos. $(p=0,020)$.

\subsubsection{Sangramento}

O volume médio de sangramento nos grupos foram respectivamente 376, 460 e 347ml, sem diferença clínica ou estatística (Gráfico 5).

Gráfico 3- Sangramento segundo escore R.E.N.A.L: baixa, média e alta complexidade

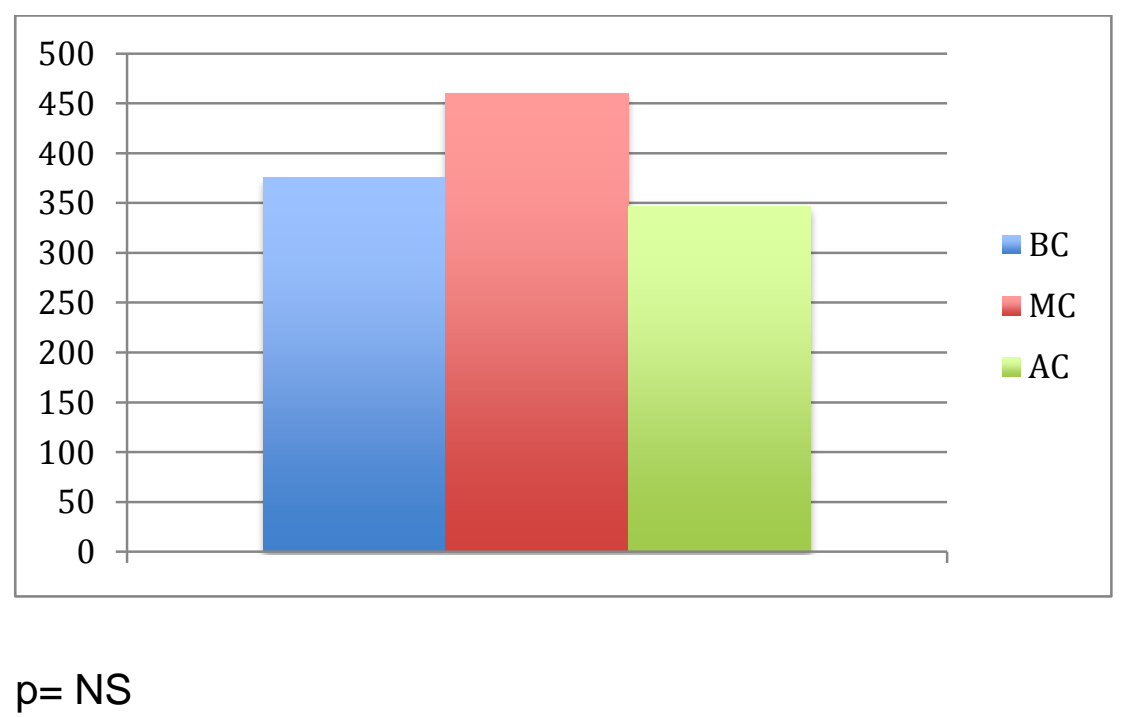

A mediana de sangramento foi de $300 \mathrm{ml}$. Quarenta por cento dos pacientes tiveram sangramento $\geq 500 \mathrm{ml}$ e $10 \% \geq 1.000 \mathrm{ml}$. Apenas 1 paciente recebeu transfusão de sangue, o volume de sangue perdido nessa cirurgia foi de 2 litros. Não encontramos correlação entre RENAL e volume de sangramento. 


\subsection{Análise da influência das variáveis nefrométricas individualmente no desfecho cirúrgico}

Analisamos individualmente a influência de cada variável do escore RENAL em predizer tempo de cirurgia, tempo de isquemia e sangramento, em modelos uni e multivariados, e obtivemos os seguintes resultados.

\subsubsection{Tempo cirúrgico}

O tamanho tumoral e a localização em relação às linhas polares foram preditores de tempo de cirurgia $\geq 180$ minutos na análise univariada. Os pacientes com tumores maiores que $4 \mathrm{~cm}$ tiveram 3,45 vezes mais chance de ter tempo de cirurgia $\geq 180$ min. $(p=0,020)$.

Do mesmo modo, encontramos que os indivíduos com tumores cruzando as linhas polares tiveram 5,5 vezes mais chance de ter tempo de cirurgia $\geq 180$ minutos do que quem tinha tumores polares $(p=0,042)$ e 3,67 vezes mais chance de ter tempo de Cirurgia $\geq 180$ minutos do que quem tinha tumor entre os polos. $(p=0,031)$.

Analisando-se as duas variáveis em modelo de regressão logística multivariada, apenas o tamanho tumoral manteve-se como fator independente influenciando o tempo de cirurgia prolongado ( $>180$ minutos) com os mesmos valores de OR obtidos na univariada (Tabelas 13). 
Tabela 13- Análise univariada dos componentes do RENAL individualmente e tempo de cirurgia

\begin{tabular}{lccc}
\hline & \multicolumn{3}{c}{ Tempo de cirurgia $>180$ min } \\
\hline & OR & $95 \%$ IC & $p$ \\
\hline $\mathrm{R}<4 \mathrm{~cm}=$ ref & & & \\
$4-7 \mathrm{~cm}$ & 3,45 & $1,2-9,8$ & 0,02 \\
\hline $\mathrm{E}>50 \%=$ ref \\
$<50 \%$ \\
Endofítico & 2,24 & $0,94-5,3$ & 0,068 \\
$\mathrm{~N} 1=$ ref & 3,5 & $0,7-4,2$ & 0,07 \\
2 & & & \\
3 & 2,3 & $0,9-5,8$ & 0,08 \\
\hline $\mathrm{A}$ (anterior = ref) & 3,1 & $0,4-4,8$ & 0,09 \\
Posterior & & & \\
$\mathrm{X}$ & 2,2 & $0,7-7,1$ & 0,2 \\
\hline $\mathrm{L}$ (polar = ref) & 2,4 & $0,6-9,0$ & 0,2 \\
Cruza a linha polar & & & \\
Entre os polos & 5,5 & $1,1-28,5$ & 0,05 \\
\hline
\end{tabular}

\subsubsection{Tempo de isquemia}

As variáveis que compõem a nefrometria RENAL individualmente não foram capazes de predizer tempo de isquemia > que 20 minutos (Tabela 14). 
Tabela 14- Análise univariada dos componentes do RENAL individualmente e tempo de isquemia

\begin{tabular}{llll}
\hline \multicolumn{4}{l}{ Tempo de isquemia $>20$ min } \\
\hline $\mathrm{R}<4 \mathrm{~cm}=$ ref & OR & $95 \%$ IC & $\mathrm{p}$ \\
$4-7 \mathrm{~cm}$ & 1,23 & $0,4-4,0$ & 0,9 \\
\hline $\mathrm{E}>50 \%=$ ref & & & \\
$<50 \%$ & 2 & $0,6-7,0$ & 0,3 \\
Endofítico & 4 & $0,3-53$ & 0,3 \\
\hline $\mathrm{N} 1=$ ref & & & 0,3 \\
2 & 1,7 & $0,5-4,0$ & 0,2 \\
3 & 2,1 & $0,3-4,5$ & 0,3 \\
\hline A (anterior = ref) & & & 0,7 \\
Posterior & 1,9 & $0,5-7,2$ & 0,6 \\
X & 1,4 & $0,3-6,6$ & 0,7 \\
\hline L (polar = ref) & 1,7 & $0,3-10$ & 0,7 \\
Cruza a linha polar & & $0,3-6$ & 0,7 \\
Entre os polos & & & \\
\hline
\end{tabular}

\subsubsection{Sangramento}

Analisando-se individualmente suas variáveis, apenas a variável $R$, que diz respeito ao tamanho do tumor, influenciou no volume de sangramento $\geq$ 500ml. Indivíduos com diâmetro tumoral maior que $4 \mathrm{~cm}$ tiveram 5,694 vezes mais chance de ter volume de sangramento $\geq 500 \mathrm{ml}$ do que os com tumores $\leq 4 \mathrm{~cm}(\mathrm{p}=0,003),($ Tabela 15). 
Tabela 15- Análise univariada dos componentes do RENAL individualmente e sangramento

\begin{tabular}{|c|c|c|c|}
\hline & \multicolumn{3}{|c|}{ Sangramento $>500 \mathrm{ml}$} \\
\hline & OR & IC & $p$ \\
\hline \multicolumn{4}{|l|}{$\mathrm{R}<4 \mathrm{~cm}=$ ref } \\
\hline $4-7 \mathrm{~cm}$ & 5,7 & $1,8-18,2$ & 0,03 \\
\hline \multicolumn{4}{|l|}{$E>50 \%=r e f$} \\
\hline$<50 \%$ & 0,9 & $0,3-2,5$ & NS \\
\hline Endofítico & 0,0 & $0,0--$ & \\
\hline \multicolumn{4}{|l|}{$\mathrm{N1}=$ ref } \\
\hline 2 & 0,4 & $0,04-2,6$ & NS \\
\hline 3 & 0,3 & $0,05-1,8$ & \\
\hline \multicolumn{4}{|l|}{ A (anterior = ref) } \\
\hline Posterior & 1,8 & $0,5-5,9$ & NS \\
\hline$x$ & 2,7 & $0,7-10,8$ & \\
\hline \multicolumn{4}{|l|}{$\mathrm{L}($ polar = ref) } \\
\hline Cruza a linha polar & 1,5 & $0,8-4,5$ & NS \\
\hline Entre os polos & 2,5 & $0,8-6,3$ & \\
\hline
\end{tabular}

\subsection{Tumores Hilares}

Apenas 5 pacientes apresentaram tumores hilares. Todos foram submetidos à nefrectomia parcial, 3 NPA e 2 NPL. Desses, 60\% tiveram margens consideradas "não livres": $40 \%$ coincidentes e $20 \%$ comprometidas, contra $4,5 \%$ dos tumores não hilares $(p=0,15)$. A presença do tumor próximo 
aos vasos do hilo renal não associou-se a piores desfechos intra-operatórios. Tabela 16.

Tabela 16- Desfechos intra operatórios e localização hilar do tumor renal

\begin{tabular}{|c|c|c|c|}
\hline & Tumor hilar & Tumor não hilar & $p$ \\
\hline Tempo cirurgia & 141,5 & 167,5 & NS \\
\hline Sangramento & 310 & 316,4 & NS \\
\hline $\begin{array}{l}\text { Tempo } \\
\text { isquemia }\end{array}$ & 14,1 & 17,6 & NS \\
\hline
\end{tabular}

\subsection{Complicações pós-operatórias}

Não houve complicações maiores relacionadas diretamente ao procedimento cirúrgico (e.g. sangramento pós-operatório, fístulas urinárias ou pseudo-aneurismas) com repercussão clínica durante o tempo de seguimento de 90 dias. As complicações medidas pelo escore de Clavien não foram verificadas no grupo de baixa complexidade, ocorreram em $7(16,6 \%)$ e em 1 $(4,5 \%)$ indivíduo nos grupos de média e alta complexidade respectivamente $(p=N S)$.

Apenas 8 pacientes $(11,3 \%)$ apresentaram complicações maiores (Clavien $\geq 3$ ). Realizou-se uma análise de regressão logística para avaliar fatores preditivos de complicações maiores pós-operatórias com as seguintes variáveis: RENAL, ASA, idade, via de acesso cirúrgica e tempo de cirurgia. Apenas ASA e idade se associou-se a complicações. Tabela 17 
Tabela 17- Variáveis preditoras de Clavien $>3$

\begin{tabular}{lccc}
\hline & OR & $95 \% \mathrm{Cl}$ & $\mathrm{P}$ \\
\hline RENAL & 0,95 & $0,6-1,5$ & 0,84 \\
ASA $>2$ & 11,48 & $1,9-69,5$ & 0,008 \\
Idade $>65$ & 1,08 & $1,1-1,2$ & 0,043 \\
Via de acesso cir. & 0,9 & $0,8-1,3$ & 0,783 \\
Tempo de cir. & 1,01 & $0,99-1,0$ & 0,1 \\
\hline
\end{tabular}

\subsection{Margens cirúrgicas}

Quatro pacientes (5,6\%) apresentaram margens cirúrgicas comprometidas. Não houve diferença entre os grupos determinados pelo escore RENAL: 1 (14,3\%); $2(4,8 \%)$; e 1 (4,5\%) nos grupos de BC, MC e AC respectivamente (Tabela 6).

Analisando-se os pacientes que tiveram margens cirúrgicas totalmente livres, ou seja, excluindo-se os casos com margens comprometidas ou coincidentes, $25 \%$ dos pacientes com tumores totalmente endofíticos tiveram margens cirúrgicas livres, contra $64,1 \%$ e $84,1 \%$ dos pacientes com tumores com menos de $50 \%$ e mais que $50 \%$ exofíticos. $(p=0,02)$. Essa frequência se manteve quando analisou-se apenas os pacientes submetidos à nefrectomia parcial: 25\%, 60\%, 84,6\%, $p=0,02$. 
5. Discussão 
O tumor renal, que já foi conhecido como tumor do internista por se apresentar mimetizando síndromes clínicas, atualmente, pode ser chamado de tumor do radiologista, pois, na maioria dos casos, é diagnosticado incidentalmente em exames de imagem(19). Em nossa casuística, encontramos $54(76 \%)$ tumores incidentais e essa frequência foi maior nos grupos de menor complexidade segundo o escore RENAL, dessa forma, os tumores que conferem algum sintoma são de estagio mais elevado e mais complexos anatomicamente e, portanto, mais difíceis de serem operados do que os não sintomáticos, sobretudo por videolaparoscopia.

A difusão e facilidade de acesso da população aos métodos de imagem permitiram diagnósticos mais precoces com lesões, em sua maioria, descobertas com tamanho menores do que $7 \mathrm{~cm}$. A nefrectomia parcial tem respaldo oncológico semelhante à nefrectomia radical para tratamento de tumores menores do que $7 \mathrm{~cm}$ (33) e acrescenta menor risco de evolução para doença renal crônica, doenças cardiovasculares e morte(15). Estes pacientes podem se beneficiar da cirurgia poupadora de néfrons. Entretanto existem outros parâmetros relacionados à anatomia do tumor, além do tamanho, que conferem dificuldade técnica à cirurgia.

Por outro lado, o advento da laparoscopia permitiu cirurgias menos mórbidas, porém mais difíceis tecnicamente, sobretudo em nefrectomias parciais. Esta cirurgia pode ser realizada com resultados oncológicos e funcionais semelhantes à aberta para tumores pequenos(17). A dificuldade técnica da nefrectomia parcial laparoscópica e as vantagens da laparoscopia, contribuem para aumentar a indicação de nefrectomia total, mesmo em lesões 
que poderiam ser ressecadas com preservação de néfrons. Entretanto, é consenso entre os urologistas que é melhor realizar uma nefrectomia parcial aberta do que radical laparoscópica(34). Considerando-se aspectos referentes à segurança oncológica, preservação da função renal e morbidade, temos como melhor escolha para pacientes com tumor renal a nefrectomia parcial videolaparoscópica, seguida pela nefrectomia parcial aberta, nefrectomia total videolaparoscópica e nefrectomia total aberta. A tomada de decisão baseada no exame de imagem pré-operatório, muitas vezes é difícil. Ultimamente vemse utilizando a nefrometria para auxiliar a conduta, porém a decisão final muitas vezes é intra-operatória.

No estudo de Patel em 2012(18), a frequência de nefrectomia parcial realizada por videolaparoscopia nos Estados Unidos foi menor do que $5 \% \mathrm{em}$ 2008, sobretudo fora das áreas urbanas e relacionadas a hospitais de ensino ou com alto volume cirúrgico. Outra avaliação realizada em centros terciários Europeus(23) encontraram aumento de 15\% para 70\% entre 1987 e 2008. Em nossa casuística, a frequência de nefrectomia parcial foi de $84,5 \%$, dessas, $53 \%$ por videolaparoscopia. Comparando-se a frequência de nefrectomia parcial do estudo piloto $(10,3 \%, 2008-2010)$ com a do estudo prospectivo $(84,5 \%, 2010$ - 2012), percebe-se um evidente aumento da sua realização em pouco tempo. Esse resultado mostra que a nossa instituição acompanhou a tendência de indicação de cirurgia preservadora de néfrons que ocorreu em outros centros de excelência e, atualmente, a realização dessa cirurgia é prioridade em nosso serviço, independentemente da via de acesso cirúrgica. 
Apesar das vantagens lógicas em se preservar néfrons, demonstrada em diversos estudos retrospectivos, o único estudo randomizado que avaliou nefrectomia parcial versus total, demonstrou resultados semelhantes em termos de sobrevida global(30). Este trabalho demonstra que a nefrectomia total não é procedimento proibitivo em indivíduos com rim contralateral normal e função renal adequada, sobretudo em tumores complexos anatomicamente que poderiam dificultar a cirurgia e aumentar as taxas de complicações ou comprometer a segurança oncológica. Possivelmente pacientes com função renal normal, rim contralateral preservado e mais idosos tenham pouco benefício da NP(35)

\subsection{Desfechos perioperatórios}

\subsubsection{Conversão}

Do ponto de vista técnico, a escolha entre nefrectomia parcial ou total ainda é muito subjetiva e, mesmo cirurgiões experientes, muitas vezes ficam em dúvida se a lesão pode ser extirpada de maneira a preservar parênquima renal funcionante e por técnica minimamente invasiva. Com a utilização da nefrometria pode-se obter parâmetros objetivos para prever taxas de conversão. Em nossa casuística, conseguimos identificar os pacientes com maiores chances de conversão. Indivíduos com escore maior ou igual a 9 estão sob elevado risco, e não seria demérito já indicar essa cirurgia por via aberta. Funahashi e cols. avaliaram retrospectivamente dados anatômicos do tumor 
renal associados à via de acesso para nefrectomia parcial e encontraram que a relação do tumor com a superfície renal ("endofiticidade") e a distância do seio renal afetou a decisão do cirurgião pela via de acesso aberta ou minimamente invasiva, e o tamanho do tumor não influenciou nessa decisão(36). Gill et al. em análise semelhante de 771 NPL e 1029 NPA reportaram que o tamanho do tumor (2.6 cm NPL vs 3.3 cm NPA) e o caráter endofítico (34.4\% NPL vs 53.3\% NPA) foram significativamente diferentes entre as duas vias de acesso.(17) Naya et al. avaliaram fatores que influenciaram a frequência de NTL (68 pacientes) vs NPL (74 pacientes). Eles encontraram que o escore RENAL até 8 foi o melhor ponto de corte para seleção de pacientes que foram submetidos à NPL.(37) Em outro trabalho, os autores compararam dados de 32 e 23 pacientes submetidos à nefrectomia parcial robótica ou aberta respectivamente, encontraram um escore médio de RENAL $>6,5$ como fator preditor significante para escolha da via de acesso aberta (38).

À medida em que esses dados forem sendo validados por estudos com casuísticas maiores, permitir-se-á uma melhor previsão das chances de conversão, melhorando o planejamento anestésico e cirúrgico e a preparação do paciente para essa possibilidade. Além disso, tumores tecnicamente favoráveis deveriam ser mais operados por NPL, sendo considerado iatrogenia uma indicação a priori diferente.

O fato de o presente estudo ter sido prospectivo reduz vieses de confusão que poderiam explicar alguns casos de conversão. Assim, excluindose da casuística pacientes com possíveis alterações anatômicas que pudessem interferir nas taxas de conversão, tais como cirurgias prévias, 
calculose renal ou pacientes com rim único, condições que sabidamente dificultam o acesso cirúrgico laparoscópico, aumentamos o poder da influência do escore RENAL neste desfecho.

\subsubsection{Tempo de cirurgia}

O tempo médio de cirurgia foi $167 \pm 54$ minutos variando de 70 a 355 minutos e foi maior conforme se aumentou os graus de complexidade segundo o escore RENAL. Ng e colaboradores(39) relataram um tempo cirúrgico de 3,5 horas em nefrectomia parcial laparoscópica. Marszalek(40) e colaboradores tiveram um tempo médio de 139 minutos. Tivemos um tempo de 174 minutos nas nefrectomias parciais VLP.

Um tempo de cirurgia maior do que 180 minutos passa a se correlacionar com complicações pós-operatórias, sobretudo pulmonares como atelectasias, hipoxemia e pneumonia(41). O escore RENAL se correlacionou com tempo de cirurgia, sendo que os indivíduos no grupo de alta complexidade tiveram um tempo cirúrgico maior, e os indivíduos com RENAL $\geq 8$ tiveram 4,94 vezes mais chances de terem tempo de cirurgia $\geq 180$ minutos. Esse dado mostrou-se altamente sensível, porém com baixa especificidade, por isso podemos afirmar que dificilmente o tempo cirúrgico de um tumor com escore RENAL <8 passará de 3 horas.

Quando analisou-se individualmente as variáveis que compõem o RENAL, apenas o tamanho tumoral e a localização em relação às linhas polares foram preditores de tempo de cirurgia $\geq 180$ minutos na análise 
univariada. Entretanto, analisando-se as duas variáveis em modelo de regressão logística multivariada, apenas o tamanho tumoral manteve-se como fator independente influenciando o tempo de cirurgia prolongado $(\geq 180$ minutos) com os mesmos valores de OR obtidos na univariada. Os indivíduos com tumores entre 4 e $7 \mathrm{~cm}$ tiveram uma chance 3,45 vezes maior de ter tempo de cirurgia $\geq 180$ minutos. Não encontramos trabalhos na literatura analisando cada componente do RENAL individualmente e tempo de cirurgia.

De acordo com essa análise, podemos concluir que apenas o tamanho do tumor oferece as informações suficientes que o cirurgião precisa para prever o tempo de cirurgia, não sendo necessário calcular o RENAL.

\subsubsection{Tempo de isquemia}

A literatura não é consensual em relação ao tempo de isquemia seguro para evitar lesão renal permanente. Existem trabalhos que demonstram que entre 20 e 30 minutos de oclusão da artéria renal não ocorre dano permanente ao tecido renal(42)(43)(44), além disso existe uma variação individual sobretudo se considerarmos a idade de incidência dos tumores renais e a prevalência de comorbidades com Hipertensão Arterial Sisitêmica e Diabete Mellitus que sabidamente contribuem para perda de função renal a longo prazo(45). Além disso a perda de parênquima renal consequente ao volume ressecado durante a nefrectomia parcial contribui para a perda de função renal(46). Adotamos um ponto de corte de 20 minutos de isquemia quente como tolerável durante as nefrectomias parciais, pois além de ser respaldado 
por vários estudos(47), alguns autores advogam que existem vantagens do menor tempo de isquemia possível(48)(49).

Encontramos um tempo mediano de isquemia de 17 e 11,5 minutos para NPL e NPA respectivamente $(p=0,07)$. O grupo da Cleveland Clinic(39) teve um tempo médio de 31 minutos em NPL. Marszalek e colaboradores(40) tiveram um tempo médio de 24 minutos. A nefrometria e o tamanho tumoral foram equivalente nestes estudos.

Evidenciamos também tempo de isquemia quente aumentou conforme os graus de complexidade tumoral segundo o escore RENAL. Vinte e um pacientes tiveram tempo de isquemia $\geq 20$ minutos. Essa proporção foi maior nos grupos de alta e média complexidades.

Encontramos que quem tem escore $\geq 9$ tem $28,6 \%$ de sensibilidade $e$ $81,6 \%$ de especificidade de ter tempo de clampeamento $>20 \mathrm{~min}$, mas a AUC foi baixa $(0,643)$, possivelmente por termos uma casuística relativamente pequena.

Mayer e Godoy, 2012,(50), encontraram que os pacientes com escore RENAL $\geq 7$ tiveram a chance aumentada de isquemia quente em 10,9 vezes. $O$ tempo médio de isquemia quente entre os grupos de baixa, media e alta complexidade foram 18, 22 e 27 minutos respectivamente. Nesse trabalho, foram analisados apenas casos operados por laparoscopia ou robô. Outros trabalhos também encontraram tempo de isquemia maiores 27,$8 ; 30,7$ e 37,9 entre os grupos(51). Uma explicação para isso é que operou-se mais indivíduos com tumores de alta complexidade $(37,4 \%)$ do que em nosso trabalho (17,9\%) (apenas nefrectomia parcial laparoscópica). 
Analisando-se individualmente as variáveis do escore RENAL, nenhuma variável foi capaz de prever tempo de isquemia quente. Encontramos um único trabalho que analisou individualmente os componentes do RENAL com isquemia quente e encontrou-se em análise multivariada que o $\mathrm{R}$ e $\circ \mathrm{N}$ se correlacionaram com tempo de isquemia(50). Concluímos que o cálculo do escore oferece mais informações do que qualquer uma de suas variáveis individualmente no que diz respeito a tempo de isquemia, em nossa amostra.

\subsubsection{Sangramento}

O adequado controle vascular e a padronização da sutura do parênquima renal permitiram aumentar a segurança das nefrectomias parciais em relação ao sangramento. Não houve correlação entre RENAL e sangramento, possivelmente pelo baixo volume de perda sanguínea da nossa amostra. A literatura mostra que, apesar de o volume de sangramento ser menor com as técnicas minimamente invasivas, a diferença clínica não é significativa, com poucos pacientes necessitando de transfusão de sangue.(52)(53)

\subsection{Margens cirúrgicas}

O escore RENAL não se correlacionou com margens cirúrgicas comprometidas, mas parece ter influência na exiguidade das mesmas. Considerando-se que o comportamento do tumor renal costuma ser não 
infiltrativo, margens coincidentes têm o mesmo prognóstico de margens negativas(54)(55)(56).

Encontramos maior número de pacientes com margens positivas no grupo operado por laparoscopia, porém sem significância estatística, em nossa casuística. A literatura demostra semelhança em relação ao comprometimento das margens em relação às vias de acesso em alguns trabalhos(57)(58)(59). Entretanto, um estudo americano com casuística do NCDB (National Cancer Database), com 11587 pacientes, relatou maior risco de margem positiva no grupos operados por técnicas minimamente invasivas em relação à cirurgia aberta: $8.1 \%$ (NPL) e $8.7 \%$ (NPR) $4.9 \%$ (NPA), com OR de 1,81 e 1,79, respectivamente, $p<0,001$.(60)

Concluímos que, em mãos experientes, a nefrectomia parcial por técnicas minimamente invasivas pode ter a mesma segurança oncológica da cirurgia aberta, porém esse dado não é reprodutível em todos os centros, portanto a NPA mantém-se como excelente opcção para tratamento de massas renais $\leq \mathrm{T} 1 \mathrm{~b}$.

\subsection{Complicações perioperatórias}

As taxas de complicações definidas como Clavien $\geq 3$ foram de $11 \%$, de acordo com o que relata a literatura que gira em torno de $6 \%$ a $20 \%(61)(62)$. Nenhum paciente apresentou fístula urinária ou sangramento. Essas complicações acontecem em aproximadamente 6,6\% dos casos(61). Não encontramos associação entre RENAL e complicações. Outros trabalhos já 
demonstraram maior incidência de fístulas urinárias. Stroup(61) mostrou correlação entre o aumento do RENAL e fístula urinária $\mathrm{OR}$ 1,56 p=0,002. Bruner e colaboradores(62) também demonstraram essa correlação. Simhan(51), em uma analise retrospectiva encontrou mais complicações no grupo de alta complexidade (OR, 5,4 Cl 1,2-24,2). Rosevear(63) identificou em analise de subgrupos que escores de RENAL mais elevados foram encontrados em pacientes com complicações maiores, mas fatores de confundimento não foram controlados neste estudo. Por outro lado, vários estudos parecem concordar não haver relação entre RENAL e outras complicações (64)(65). Conclui-se que o uso do escore RENAL para prever complicações é controverso.

Não houve, em nossa casuística, diferença significativa entre as diferentes vias de acesso e complicações. Outros trabalhos também não encontraram diferenças entre as cirurgias minimamente invasivas (robótica ou VLP) e aberta(61)(53)(66)(52)(67).

Não houve casos de óbito em nossos pacientes. A mortalidade publicada é por volta de $0,5 \%(68)$.

\subsection{Análise crítica das variáveis que compõem o RENAL}

\subsubsection{RADIUS}

Encontramos que o tamanho do tumor foi fator de risco independente para tempo de cirurgia e isquemia prolongados. $O$ tamanho do tumor 
influencia na dificuldade técnica de abordagem cirúrgica por dificultar a exposição e manipulação do massa tumoral, além de supor um comportamento biológico mais agressivo, como demonstram alguns trabalhos(51)(29). Entretanto não houve diferença entre os subtipos histológicos e o grau de Furhman com relação ao tamanho do tumor, possivelmente porque abordamos apenas lesões $\leq 7 \mathrm{~cm}$.

\subsubsection{Exophyticus/Endophyticus}

A relação do tumor com a superfície renal influencia na quantidade e profundidade de secção do parênquima renal promovendo lesão de estruturas vasculares mais calibrosas e sistema coletor. Influencia também no planejamento da ressecção no que diz respeito à margem tumoral, pois quanto mais endofítico o tumor, mais difícil o planejamento prévio da ressecção e teoricamente maior o risco de sangramento, fistulas e margem comprometida. (69)

Não encontramos maior sangramento ou fístula urinária, porém $25 \%$ dos pacientes com tumores totalmente endofíticos tiveram margens cirúrgicas livres, contra 64,1 e $84,1 \%$ dos pacientes com tumores com menos de $50 \%$ e mais que $50 \%$ exofíticos. Em tumores totalmente endofíticos a ressecção requer auxílio de ultrassonografia, ou em casos de cirurgia aberta, uma nefrotomia anatrófica pode ser realizada.(70) Possivelmente a relação do 
tumor com o parênquima renal é um dos fatores que compõem o escore RENAL que mais influencia na dificuldade técnica do procedimento.

\subsubsection{Nearness}

A proximidade do tumor com o sistema coletor e seio renal também confere maiores riscos de lesões vasculares e de sistema coletor, determinando maior chance de sangramento e fístulas urinárias. Entretanto, não encontramos correlação desta variável com desfechos perioperatórios ou complicações. Mayer e Godoy, 2012 (50)analisaram a influência do componente $\mathrm{N}$ do escore RENAL e complicações perioperatórias e encontraram diferença entre os grupos N1, 2 ou 3 no tempo de isquemia (18; $22 ; 27 \min , p<0,01)$.

\subsubsection{Anterior/Posterior}

Os tumores de face posterior de rim, ao serem abordados por laparoscopia transperitoneal, necessitam de amplo deslocamento do rim e conferem maior dificuldade de ressecção e sutura do parênquima, podendo aumentar o tempo cirúrgico, de isquemia e sangramento. Ate o momento, nenhum trabalho comentou essa impressão compartilhada pelos cirurgiões laparoscópicos. Nenhuma nefrometria até o momento atribui pontos a essa observação. 
Em nossa casuística, 17 pacientes(23,9\%) tinham tumores localizados na face posterior do rim. Estes foram menos operados por laparoscopia, apesar de a diferença não ter sido significante, provavelmente devido ao pequeno número de pacientes na análise, acreditamos que houve uma preferência por parte dos cirurgiões a aborda-los de maneira aberta.

Das variáveis que sugerem maior dificuldade técnica durante a cirurgia, apenas o sangramento mostrou-se significante, sendo maior nas cirurgias de tumores de localização posterior $(629 \mathrm{ml} \times 360 \mathrm{ml}$, p: 0,025$)$, para a via laparoscópica e aberta respectivamente, entretanto, sem diferenças entre o tempo de isquemia (18 min x $18 \mathrm{~min}$ ) ou tempo cirúrgico (182 min x $162 \mathrm{~min}$, NS).

\subsubsection{Latitude}

O posicionamento do tumor em relação às linhas polares conforme proposto por Uzzo(24), torna o procedimento mais difícil apenas se vier associado a outros componentes do RENAL com lesões grandes, endofíticas ou hilares. Um tumor entre as linhas polares, pequeno, exofítico e em face anterior de rim é tão fácil de ressecar quanto uma lesão semelhante em polo inferior. Entretanto, acreditamos que os tumores de polo superior conferem mais dificuldade à laparoscopia, pois costumam ter relação com outros órgãos como fígado, baço ou cauda do pâncreas e posicionam-se opostamente à via de acesso, dificultando a ressecção e sutura do parênquima renal. Entretanto, 
nossos dados não confirmaram essa hipótese, pois não conseguimos encontrar diferença nos desfechos perioperatórios (sangramento, tempo de isquemia ou cirurgia, complicações) dos tumores de polo superior em relação aos de outras topografias.

\subsubsection{Hilar}

Os tumores hilares, que encostam nos vasos principais do hilo (artéria ou veia renais ou segmentares), sua ressecção confere maior complexidade pelo risco de lesão desses vasos e consequente sangramento ou isquemia de áreas de parênquima saudável. Muitas vezes é necessário totalização da cirurgia. Não encontramos trabalhos na revisão bibliográfica que relacionasse tumores hilares ao maior risco de nefrectomia total. No trabalho original que propõe o RENAL(24), os autores não conferem pontuação aos tumores hilares, mas apenas acrescentam o sufixo "H" e o tumor é classificado como de alta complexidade, independentemente do escore.

Acreditamos que esses tumores hilares pequenos são, em sua maioria, ressecáveis, pois, apesar de encostarem nos vasos, não costumam invadi-los ou causarem aderências fixas. Entretanto, a necessidade de dissecção muito próxima à cápsula tumoral aumenta a chance de margens comprometidas. Dos cinco pacientes com tumores hilares, $60 \%$ tiveram margens consideradas "não livres": $40 \%$ coincidentes e $20 \%$ comprometidas, contra $4,5 \%$ dos tumores não 
hilares. Não encontramos associações com desfechos intra-operatórios (tempo de isquemia, tempo de cirurgia ou sangramento).

Deve-se ressaltar que o número reduzido de pacientes com tumores hilares enfraqueceu o poder dessas análises.

\subsubsection{Conclusões acerca da análise crítica das variáveis que compõem o RENAL}

Concluímos com essa análise que algumas variáveis do RENAL não influenciam desfechos, como a latitude (L), e outras são redundantes, como $\mathrm{E}$ e N. Por outro lado, o R e o E são mais importantes e deveriam receber pesos maiores na pontuação do escore. Sugerimos a construção de um escore ponderado de acordo com a influência de cada uma das variáveis.

Recentemente foi proposto pelo grupo da Universidade do Sul da Califórnia (69) o cálculo da área da superfície de contato do tumor com o parênquima renal. Um novo método, calculado através da tomografia, que permite classificar a complexidade anatômica do tumor renal. Seria uma tentativa de reduzir a redundância de algumas variáveis do RENAL e privilegiar tamanho e relação do tumor com o parênquima renal, justamente as duas variáveis que foram mais relevantes no nosso estudo. Esse novo escore se correlacionou com desfechos perioperatórios, porém necessita de mais estudos para sua validação. 


\subsection{Limitações do estudo}

O presente estudo, apesar de prospectivo, não controlou algumas variáveis. A variação das equipes cirúrgicas pode ter influenciado na escolha da via de acesso, apesar da formação homogênea dos médicos do ICESP. Outro aspecto negativo foi a perda amostral e o número reduzido de pacientes para realização de algumas análises, o que pode ter reduzido o poder dos testes estatísticos. Foram excluídos indivíduos com fatores que pudessem influenciar nos desfechos perioperatórios como cirurgia renal previa ipsilateral, calculose renal ou cirurgia em andar superior de abdome prévio, isso reduziu muito a casuística. Esse fato também pode explicar os melhores resultados obtidos em nossa amostra em relação ao publicado por grandes centros de referência no que diz respeito a tempo de cirurgia, isquemia e complicações. 
6. Conclusões 
1. O escore RENAL pode ser usado para prever chances de conversão e totalização da nefrectomia, tempo de clampeamento e tempo de cirurgia.

2. Os dados tomográficos mais importantes do escore, de acordo com essa casuística, foram tamanho tumoral $(R)$ e relação com a superfície renal (E). 
7. Anexos 


\section{Anexo 1- $\quad$ Aprovação CAPPesq}

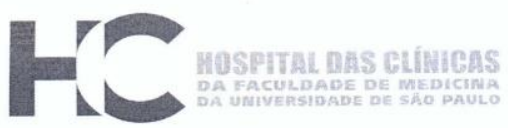

\section{APROVAÇĀO}

A Comissão de Ética para Análise de Projetos de Pesquisa CAPPesa da Diretoria Clínica do Hospital das Clínicas da Faculdade de Medicina da Universidade de São Paulo, em sessão de 02/02/2011, APROVOU o Protocolo de Pesquisa $n^{\circ}$ 0027/11, intitulado: "FATORES TOMOGRÁFICOS PREDITIVOS DE DESFECHOS CIRÚRGICOS APÓS NEFRECTOMIA PARCIAL: APLICAÇĀO PROSPECTIVA DO ESCORE RENAL" apresentado pelo Departamento de CIRURGIA, inclusive O Termo de Consentimento Livre e Esclarecido.

Cabe ao pesquisador elaborar e apresentar à CAPPesq, os relatórios parciais e final sobre a pesquisa (Resolução do Conselho Nacional de Saúde n० 196, de 10/10/1996, inciso (X.2, letra "c").

Pesquisador (a) Responsável: Marcos Francisco Dall'Oglio

Pesquisador (a) Executante: Andre Costa Matos

CAPPesq, 03 de Fevereiro de 2011
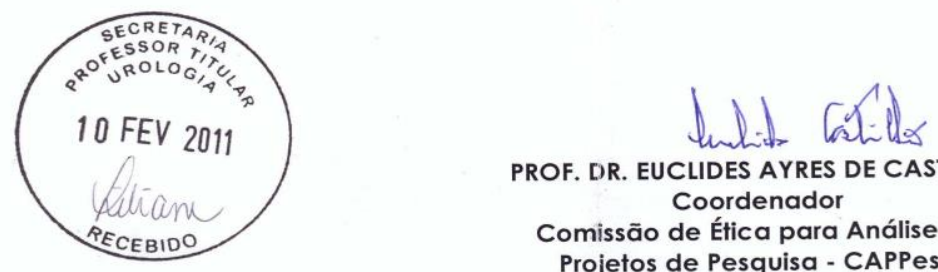

PROF. DR. EUCLIDES AYRES DE CASTILHO Coordenador

Comissão de Ética para Análise de Projetos de Pesquisa - CAPPesq

Comissăo de Ética para Análise de Projetos de Pesquisa do HCFMUSP da Diretoria Clínica do Hospital das Clinicas da Faculdade de Medicina da Universidade de São Paulo Rua Ovídio Pires de Campos, 225, $5^{\circ}$ andar - CEP 05403010 - São Paulo - SP Fone: 01130696442 Fax: 01130696492 e-mail: cappesq@hcnet.usp.br 


\section{Anexo 2- $\quad$ Termo de Consentimento Livre e Esclarecido}

\section{TERMO DE CONSENTIMENTO LIVRE E ESCLARECIDO}

Prezado(a) participante:

Sou médico do Serviço de Urologia do Instituto do Câncer do Estado de São Paulo. Estou realizando uma pesquisa sob supervisão do professor Marcos Dall'Oglio, cujo objetivo é avaliar o papel da tomografia computadorizada na cirurgia de nefrectomia parcial (retirada parcial do rim) no tratamento do câncer renal.

Sua participação envolve responder a um questionário, colher exames de sangue e urina e realizar uma tomografia do abdome. Todos esses exames já seriam realizados normalmente para o seu tratamento, ou seja, a participação na pesquisa não mudaria em nada a sua rotina ou o seu tratamento neste serviço.

A participação nesse estudo é voluntária e se você decidir não participar ou quiser desistir de continuar em qualquer momento, tem absoluta liberdade de fazê-lo.

$\mathrm{Na}$ publicação dos resultados desta pesquisa, sua identidade será mantida no mais rigoroso sigilo. Serão omitidas todas as informações que permitam identificá-lo(a).

Mesmo não tendo benefícios diretos em participar, indiretamente você estará contribuindo para a compreensão do fenômeno estudado e para a produção de conhecimento científico.

Quaisquer dúvidas relativas à pesquisa poderão ser esclarecidas pelo(s) pesquisador(es) ou médicos do serviço de urologia.

Atenciosamente 
Anexos 68

Nome e assinatura do professor supervisor/orientador e CRM

Concordo em participar deste estudo

Nome e assinatura do paciente 
8. Referências Bibliográficas 
1. Herr HW. Partial nephrectomy for unilateral renal carcinoma and a normal contralateral kidney: 10-year follow up. J Urol [Internet]. 1999 Jan;161(1):33-5. Available from: http://linkinghub.elsevier.com/retrieve/pii/S0022534701620524

2. Uzzo RG, Novick AC. Review Article Nephron sparing surgery for renal tumors: indications, techniques and outcomes. J Urol. 2001;166(July):618.

3. Licht MR, Novick AC. Nephron sparing surgery for renal cell carcinoma. J Urol [Internet]. 1993 Jan;149(1):1-7. Available from: http://www.ncbi.nlm.nih.gov/pubmed/8417184

4. Moll V, Becht E, Ziegler M. Kidney preserving surgery in renal cell tumors: indications, techniques and results in 152 patients. J Urol [Internet]. 1993 Aug;150(2 Pt 1):319-23. Available from: http://www.ncbi.nlm.nih.gov/pubmed/8326552

5. Hafez KS, Fergany AF, Novick AC. Nephron sparing surgery for localized renal cell carcinoma: impact of tumor size on patient survival, tumor recurrence and TNM staging. J Urol [Internet]. 1999 Dec;162(6):1930-3. Available from: http://www.ncbi.nlm.nih.gov/pubmed/10569540

6. Barbalias GA, Liatsikos EN, Tsintavis A, Nikiforidis G. Adenocarcinoma of the kidney: nephron-sparing surgical approach vs. radical nephrectomy. $J$ Surg Oncol [Internet]. 1999 Nov;72(3):156-61. Available from: http://www.ncbi.nlm.nih.gov/pubmed/10562362

7. Filipas D, Fichtner JAN, Spix C, Black P, Carus W. Nephron-sparing surgery of renal cell. Urology. 2000;4295(00):387-92.

8. Delakas D, Karyotis I, Daskalopoulos G, Terhorst B, Lymberopoulos S, Cranidis A. Nephron-sparing surgery for localized renal cell carcinoma with a normal contralateral kidney: a European three-center experience. Urology [Internet]. 2002;60(6):998-1002. Available from: http://www.ncbi.nlm.nih.gov/pubmed/12475657 
9. Lane BR, Gill IS. 7-Year Oncological Outcomes After Laparoscopic and Open Partial Nephrectomy. J Urol [Internet]. Elsevier Inc.; 2010;183(2):473-9. Available from: http://dx.doi.org/10.1016/j.juro.2009.10.023

10. Matin SF, Gill IS, Worley S, Novick AC. Outcome of laparoscopic radical and open partial nephrectomy for the sporadic $4 \mathrm{~cm}$. or less renal tumor with a normal contralateral kidney. J Urol [Internet]. 2002 Oct;168(4 Pt 1):1356-60. Available from: http://www.ncbi.nlm.nih.gov/pubmed/12352392

11. Huang WC, Levey AS, Serio AM, Snyder M, Vickers AJ, Raj G V, et al. Chronic kidney disease after nephrectomy in patients with renal cortical tumours: a retrospective cohort study. Lancet Oncol [Internet]. 2006;7(9):735-40. Available from: http://linkinghub.elsevier.com/retrieve/pii/S1470204506708038

12. Thompson RH, Siddiqui S, Lohse CM, Leibovich BC, Russo P, Blute ML. Partial Versus Radical Nephrectomy for 4 to $7 \mathrm{~cm}$ Renal Cortical Tumors. J Urol [Internet] 2009;182(6):2601-6. Available from: http://dx.doi.org/10.1016/j.juro.2009.08.087

13. Thompson RH, Boorjian SA, Lohse CM, Leibovich BC, Kwon ED, Cheville $\mathrm{JC}$, et al. Radical nephrectomy for $\mathrm{pT} 1 \mathrm{a}$ renal masses may be associated with decreased overall survival compared with partial nephrectomy. $J$ Urol. 2008;179(2):468-71.

14. Capitanio U, Terrone C, Antonelli A, Minervini A, Volpe A, Furlan M, et al. Nephron-sparing Techniques Independently Decrease the Risk of Cardiovascular Events Relative to Radical Nephrectomy in Patients with a T1a - T1b Renal Mass and Normal Preoperative Renal Function. Eur Urol [Internet]. European Association of Urology; 2015;67(4):683-9. Available from: http://dx.doi.org/10.1016/j.eururo.2014.09.027 
15. Huang WC, Elkin EB, Levey AS, Jang TL, Russo P. Partial Nephrectomy Versus Radical Nephrectomy in Patients With Small Renal Tumors-Is There a Difference in Mortality and Cardiovascular Outcomes? J Urol. 2009;181(1):55-62.

16. Gill IS, Matin SF, Desai MM, Kaouk JH, Steinberg A, Mascha E, et al. Comparative analysis of laparoscopic versus open partial nephrectomy for renal tumors in 200 patients. J Urol [Internet]. 2003 Jul;170(1):64-8. Available from: http://www.ncbi.nlm.nih.gov/pubmed/12796646

17. Gill IS, Kavoussi LR, Lane BR, Blute ML, Babineau D, Colombo JR, et al. Comparison of 1,800 Laparoscopic and Open Partial Nephrectomies for Single Renal Tumors. J Urol. 2007;178(1):41-6.

18. Patel SG, Penson DF, Pabla B, Clark PE, Cookson MS, Chang SS, et al. National trends in the use of partial nephrectomy: A rising tide that has not lifted all boats. J Urol [Internet]. Elsevier Inc.; 2012;187(3):816-21. Available from: http://dx.doi.org/10.1016/j.juro.2011.10.173

19. Dall'oglio MF, Crippa A, Camara C, Pontes-Junior J, Colombo JR, Nesrallah AJ, et al. The beginning of the 21st century: a paradigm shift in the surgical management of renal cell carcinoma in South America. Int Braz J Urol. 1945;36(6):670-7.

20. Kane CJ, Mallin K, Ritchey J, Cooperberg MR, Carroll PR. Renal cell cancer stage migration: Analysis of the National Cancer Data Base. Cancer. 2008;113(1):78-83.

21. Shin SJ, Ko KJ, Kim TS, Ryoo HS, Sung HH, Jeon HG, et al. Trends in the Use of Nephron-Sparing Surgery over 7 Years: An Analysis Using the R.E.N.A.L. Nephrometry Scoring System. PLoS One [Internet]. 2015;10(11):e0141709. Available from: http://dx.plos.org/10.1371/journal.pone.0141709 
22. Zini L, Patard JJ, Capitanio U, Mejean A, Villers A, de La Taille A, et al. The use of partial nephrectomy in European tertiary care centers. Eur $\mathrm{J}$ Surg Oncol [Internet]. 2009 Jun;35(6):636-42. Available from: http://www.ncbi.nlm.nih.gov/pubmed/18775626

23. Meyer C, Hansen J, Becker A, Schmid M, Pradel L, Strini K, et al. The Adoption of Nephron-Sparing Surgery in Europe - A Trend Analysis in Two Referral Centers from Austria and Germany. Urol Int [Internet]. 2015 Dec 24; Available from: http://www.ncbi.nlm.nih.gov/pubmed/26699625

24. Kutikov A, Uzzo RG. The R.E.N.A.L. Nephrometry Score: A Comprehensive Standardized System for Quantitating Renal Tumor Size, Location and Depth. J Urol [Internet]. American Urological Association; 2009;182(3):844-53. Available from: http://linkinghub.elsevier.com/retrieve/pii/S0022534709011756

25. Ficarra V, Novara G, Secco S, Macchi V, Porzionato A, De Caro R, et al. Preoperative Aspects and Dimensions Used for an Anatomical (PADUA) Classification of Renal Tumours in Patients who are Candidates for Nephron-Sparing Surgery. Eur Urol [Internet]. 2009;56(5):786-93. Available from: http://linkinghub.elsevier.com/retrieve/pii/S030228380900788X

26. Simmons MN, Ching CB, Samplaski MK, Park CH, Gill IS. Kidney Tumor Location Measurement Using the C Index Method. J Urol [Internet]. Elsevier Inc.; 2010;183(5):1708-13. Available from: http://dx.doi.org/10.1016/j.juro.2010.01.005

27. Kolla SB, Spiess PE, Sexton WJ. Interobserver Reliability of the RENAL Nephrometry Scoring System. URL [Internet]. Elsevier Inc.; 2011;78(3):592-4. Available from: http://dx.doi.org/10.1016/j.urology.2011.05.023

28. Hayn MH, Schwaab T, Underwood W, Kim HL. BJU INTERNATIONAL outcomes of laparoscopic partial nephrectomy. 2010;876-81. 
29. Kutikov A, Smaldone MC, Egleston BL, Manley BJ, Canter DJ, Simhan J, et al. Platinum Priority - Kidney Cancer Anatomic Features of Enhancing Renal Masses Predict Malignant and High-Grade Pathology: A Preoperative Nomogram Using the RENAL Nephrometry Score. Eur Urol. 2011;60:241-8.

30. Scosyrev E, Messing EM, Sylvester R, Campbell S, Van Poppel H. Renal function after nephron-sparing surgery versus radical nephrectomy: Results from EORTC randomized trial 30904. Eur Urol [Internet]. European Association of Urology; 2014;65(2):372-7. Available from: http://dx.doi.org/10.1016/j.eururo.2013.06.044

31. Charlson ME, Pompei $\mathrm{P}$, Ales $\mathrm{KL}$ MC. A new method of classifying prognostic comorbidity in longitudinal studies: development and validation. J Chronic Dis. 1987;40(5):373-83.

32. Dindo D, Demartines N, Clavien P. Classification of Surgical Complications. Ann Surg. 2004;240(2):205-13.

33. Mason RJ, Rendon RA. Partial Nephrectomy for T1b Renal Cell Carcinoma: A Safe and Superior Treatment Option. Can Urol Assoc J. 2012;6(2):128-30.

34. Cozar JM, Tallada M. Open Partial Nephrectomy in Renal Cancer: A Feasible Gold Standard Technique in All Hospitals. Adv Urol. 2008;2008(Algorithm 1).

35. Surgery N, Woldu SL, Weinberg AC, Korets R, Ghandour R, Danzig MR, et al. Who Really Benefits From Nephron-sparing Surgery? Urology [Internet]. Elsevier Inc.; 2014;84(4):860-8. Available from: http://dx.doi.org/10.1016/j.urology.2014.05.061

36. Funahashi Y, Murotani K, Yoshino Y, Sassa N, Ishida S GM. The renal tumor morphological characteristics that affect surgical planning for laparoscopic or open partial nephrectomy. Nagoya J Med Sci. 2015;77(12):229-35. 
37. Naya $\mathrm{Y}$, Kawauchi A, Oishi M, Ueda T. Comparison of diameter-axialpolar nephrometry and RENAL nephrometry score for treatment decisionmaking in patients with small renal mass. Int J Clin Oncol. 2015;358-61.

38. Acar Ö, Musao A, Vural M. Morphometric profile of the localised renal tumors managed either by open or robot-assisted nephron-sparing surgery: the impact of scoring systems on the decision making process. BMC Urol. 2013;

39. Ng CS, Gill IS, Ramani AP, Steinberg AP, Spaliviero M, Abreu SC, et al. Transperitoneal versus retroperitoneal laparoscopic partial nephrectomy: patient selection and perioperative outcomes. J Urol. 2005;174(September):846-9.

40. Marszalek M, Chromecki T, Al-ali BM, Meixl H, Madersbacher S, Jeschke $\mathrm{K}$, et al. Comparison of the Transperitoneal Versus the Retroperitoneal Approach. Urology [Internet]. Elsevier Inc.; 2011;77(1):109-13. Available from: http://dx.doi.org/10.1016/j.urology.2010.02.057

41. Smetana GW, Lawrence VA, Cornell JE. Annals of Internal Medicine Clinical Guidelines Preoperative Pulmonary Risk Stratification for Noncardiothoracic Surgery : Systematic Review for the American College of Physicians. Ann Intern Med. 2006;

42. Secin FP. Importance and Limits of Ischemia in Renal Partial Surgery: Experimental and Clinical Research. Adv Urol. 2008;2008.

43. Desai MM1, Gill IS, Ramani AP, Spaliviero M, Rybicki L KJ. The impact of warm ischaemia on renal function after laparoscopic partial nephrectomy. U Int. 2005;95(3):377-83.

44. Becker F, Poppel H Van, Hakenberg OW, Stief C, Gill I, Guazzoni G, et al. Assessing the Impact of Ischaemia Time During Partial Nephrectomy. Eur Urol. 2009;56:625-35. 
45. Hsu C-Y, McCulloch CE, Darbinian J, Go AS IC. Elevated blood pressure and risk of end-stage renal disease in subjects without baseline kidney disease. Arch Intern Med. 2005;165(8):923-8.

46. Demirtas A, Baydilli N, Sahin N, Ekmekcioglu O, Demirci D. Assessment of Warm and Cold Ischem I a on Functions of the Operated Kidney with 99m Tc-DMSA in Renal Masses: A Prospective and Randomized Study *. Open J Urol. 2013;2013(May):62-7.

47. Thompson RH, Frank I, Lohse CM, Saad IR, Fergany A, Zincke H, et al. The Impact of Ischemia Time During Open Nephron Sparing Surgery on Solitary Kidneys: A Multi-Institutional Study. J Urol. 2007;177(February):471-6.

48. Desai MM, Luis A, Abreu DC, Leslie S, Cai J, Huang EY, et al. Robotic Partial Nephrectomy with Superselective Versus Main Artery Clamping: A Retrospective Comparison. Eur Urol [Internet]. European Association of Urology; 2014;66(4):713-9. Available from: http://dx.doi.org/10.1016/j.eururo.2014.01.017

49. Satkunasivam R, Tsai S, Syan S, Bernhard J, Luis A, If TD, et al. Surgery in Motion Robotic Unclamped " Minimal-margin " Partial Nephrectomy: Ongoing Refinement of the Anatomic Zero-ischemia Concept. Eur Urol [Internet]. European Association of Urology; 2015;68(4):705-12. Available from: http://dx.doi.org/10.1016/j.eururo.2015.04.044

50. Mayer WA, Godoy G, Choi JM, Goh AC, Bian SX, Link RE. Higher RENAL Nephrometry Warm Ischemia Time and Collecting. Urology [Internet]. Elsevier Inc.; 2012;79(5):1052-6. Available from: http://dx.doi.org/10.1016/j.urology.2012.01.048

51. Simhan J, Smaldone MC, Tsai KJ, Canter DJ, Li T, Kutikov A, et al. Objective Measures of Renal Mass Anatomic Complexity Predict Rates of Major Complications Following Partial Nephrectomy. Eur Urol. 2011;60:724-30. 
52. Porpiglia AF, Mari A, Bertolo R, Bianchi G, Fidanza F, Fiori C, et al. Partial Nephrectomy in Clinical T1b Renal Tumors: Multicenter Comprative Study of Open, Laparoscopic and Robotic-Assisted Approach (the RECORD Project). Urology [Internet]. Elsevier Inc.; 2015;(2016). Available from: http://dx.doi.org/10.1016/j.urology.2015.08.049

53. Zhang X, Shen Z, Zhong S, Zhu Z, Wang X. Comparison of peri-operative outcomes of robot-assisted vs laparoscopic partial nephrectomy : a metaanalysis. BJU Int. 2013;1133-42.

54. Minervini A, Ficarra V, Rocco F, Antonelli A, Bertini R, Carmignani G, et al. Simple Enucleation is Equivalent to Traditional Partial Nephrectomy for Renal Cell Carcinoma: Results of a Nonrandomized, Retrospective , Comparative Study. J Urol [Internet]. American Urological Association Education and Research, Inc.; 2011;185(5):1604-10. Available from: http://dx.doi.org/10.1016/j.juro.2010.12.048

55. Longo N, Minervini A, Antonelli A, Bianchi G. Simple enucleation versus standard partial nephrectomy for clinical T1 renal masses: Perioperative outcomes based on a matched-pair comparison of 396 patients ( RECORd project ). Eur J Surg Oncol [Internet]. Elsevier Ltd; 2014;40(6):762-8. Available from: http://dx.doi.org/10.1016/j.ejso.2014.01.007

56. Sundaram V, Figenshau RS, Roytman TM, Kibel AS, lii RLG, Bullock A, et al. Partial Nephrectomy: Does Cancer Remain in teh Renal Remnant. Uology [Internet]. Elsevier Inc.; 2011;77(6):1400-3. Available from: http://dx.doi.org/10.1016/j.urology.2010.12.016

57. Krane LS1 HA. Robotic and laparoscopic partial nephrectomy for T1b tumors. Curr Opin Urol 6320d2. 2013;23(5):418-22.

58. Borghesi M, Schiavina R, Gan M, Novara G, Mottrie A. Expanding utilization of robotic partial nephrectomy for clinical T1b and complex T1a renal masses. World J Urol. 2013;499-504. 
59. Volpe A1, Amparore D MA. Treatment outcomes of partial nephrectomy for T1b tumours. Curr Opin Urol. 2013;23(5):403-10.

60. Tabayoyong W, Abouassaly R, Kiechle JE, Cherullo EE, Meropol NJ, Shah ND, et al. Adult Urology Oncology: Adrenal / Renal / Upper Tract / Bladder Variation in Surgical Margin Status by Surgical Approach among Patients Undergoing Partial Nephrectomy for Small Renal Masses. J Urol [Internet]. Elsevier Ltd; 2015;194(6):1548-53. Available from: http://dx.doi.org/10.1016/j.juro.2015.06.076

61. Stroup SP, Palazzi K, Kopp RP, Mehrazin R, Santomauro M, Cohen SA, et al. RENAL Nephrometry Score is Associated With Operative Approach for Partial Nephrectomy and Urine Leak. Urology [Internet]. Elsevier Inc.; 2012;80(1):151-6. Available from: http://dx.doi.org/10.1016/j.urology.2012.04.026

62. Bruner B, Breau RH, Lohse CM, Leibovich BC, Blute ML. Renal nephrometry score is associated with urine leak after partial nephrectomy. BJUI. 2010;67-72.

63. Rosevear HM, Gellhaus PT, Lightfoot AJ, Kresowik TP, Joudi FN, Tracy CR. Utility of the RENAL nephrometry scoring system in the real world: predicting surgeon operative preference and complication risk. BJUI. 2011;700-5.

64. Nadu A, Kleinmann N, Laufer M, Dotan Z, Winkler H, Ramon J. Laparoscopic Partial Nephrectomy for Central Tumors: Analysis of Perioperative Outcomes and Complications. J Urol. 2009;181(January):42-7.

65. Frank I, Colombo JR, Rubinstein M, Desai M, Kaouk J, Gill IS. Oncology : Adrenal / Renal / Upper Tract / Bladder for Centrally Located Renal Tumors. J Urol. 2006;175(March):849-52. 
66. Masson-lecomte A, Bensalah K, Seringe E, Vaessen C, Taille A De, Doumerc N. A prospective comparison of surgical and pathological outcomes obtained after robot-assisted or pure laparoscopic partial nephrectomy in moderate to complex renal tumours: results from a French multicentre collaborative study. BJU Int. 2012;4:256-63.

67. Minervini A, Siena G, Antonelli A, Bianchi G, Simeone C, Terrone C, et al. Open versus laparoscopic partial nephrectomy for clinical T1a renal masses: a matched-pair comparison of 280 patients with TRIFECTA outcomes ( RECORd Project ). World J urol. 2014;257-63.

68. Schmitges J1, Trinh QD, Sun M, Hansen J, Bianchi M, Jeldres C, Perrotte P, Dahlem R, Shariat SF, Chun FK, Montorsi F, Menon M, Fisch M, Graefen M KP. Higher perioperative morbidity and in-hospital mortality in patients with end-stage renal disease undergoing nephrectomy for nonmetastatic kidney cancer: a population-based analysis. BJU Int. 2012;110(6 PtB):183-90.

69. Leslie S, Gill IS, Luis A, Abreu DC, Rahmanuddin S, Gill KS, et al. Renal Tumor Contact Surface Area: A Novel Parameter for Predicting Complexity and Outcomes of Partial Nephrectomy. Eur Urol [Internet]. European Association of Urology; 2014;66(5):884-93. Available from: http://dx.doi.org/10.1016/j.eururo.2014.03.010

70. Dall'Oglio MF, Ballarotti L, Passerotti CC, Paluello D V, Colombo JR, Crippa A, et al. Anatrophic Nephrotomy as Nephron-Sparing Approach for Complete Removal of Intraparenchymal Renal Tumors. Int braz j urol. 2012;38(3):356-61. 